\title{
Outsourced Symmetric Private Information Retrieval
}

\author{
Stanislaw Jarecki* Charanjit Jutla ${ }^{\dagger} \quad$ Hugo Krawczyk $^{\ddagger} \quad$ Marcel Rosu $^{\S}$ Michael Steiner
}

\begin{abstract}
In the setting of searchable symmetric encryption (SSE), a data owner $\mathcal{D}$ outsources a database (or document/file collection) to a remote server $\mathcal{E}$ in encrypted form such that $\mathcal{D}$ can later search the collection at $\mathcal{E}$ while hiding information about the database and queries from $\mathcal{E}$. Leakage to $\mathcal{E}$ is to be confined to well-defined forms of data-access and query patterns while preventing disclosure of explicit data and query plaintext values. Recently, Cash et al. presented a protocol, OXT, which can run arbitrary boolean queries in the SSE setting and which is remarkably efficient even for very large databases.

In this paper we investigate a richer setting in which the data owner $\mathcal{D}$ outsources its data to a server $\mathcal{E}$ but $\mathcal{D}$ is now interested to allow clients (third parties) to search the database such that clients learn the information $\mathcal{D}$ authorizes them to learn but nothing else while $\mathcal{E}$ still does not learn about the data or queried values as in the basic SSE setting. Furthermore, motivated by a wide range of applications, we extend this model and requirements to a setting where, similarly to private information retrieval, the client's queried values need to be hidden also from the data owner $\mathcal{D}$ even though the latter still needs to authorize the query. Finally, we consider the scenario in which authorization can be enforced by the data owner $\mathcal{D}$ without $\mathcal{D}$ learning the policy, a setting that arises in court-issued search warrants.

We extend the OXT protocol of Cash et al. to support arbitrary boolean queries in all of the above models while withstanding adversarial non-colluding servers $(\mathcal{D}$ and $\mathcal{E})$ and arbitrarily malicious clients, and while preserving the remarkable performance of the protocol.
\end{abstract}

\section{Introduction}

Consider a database DB composed of collection of documents or records and an application that needs to search DB based on the keywords contained in these records. For example, DB

*U. California Irvine. Email: stasio@ics.uci.edu.

${ }^{\dagger}$ IBM Research. Email: csjutla@us.ibm.com

${ }^{\ddagger}$ IBM Research. Email: hugo@ee.technion.ac.il

${ }^{\S}$ U. California Irvine. Email: marcelrosu@gmail.com

`IBM Research. Email: msteiner@us.ibm.com 
can be a medical relational database with records indexed by a set of attributes (e.g., name, zipcode, medical condition, etc.), an email repository indexed by English words and/or envelope information (date, sender, receivers, etc.), a collection of webpages indexed by text and metadata, etc. A search query consists of a boolean expression on keywords that returns all documents whose associated keywords satisfy that expression. In this paper we are concerned with applications where the database is outsourced to an external server $\mathcal{E}$ and search is performed at $\mathcal{E}$ privately. That is, $\mathcal{E}$ stores an encrypted version of the original database DB (plus some metadata) and answers encrypted queries from clients such that the client obtains the documents matching his query without $\mathcal{E}$ learning plaintext information about the data and queries.

The most basic setting of private data outsourcing as described above is where the owner of the data itself, $\mathcal{D}$, is the party performing the search at $\mathcal{E}$. In this setting, $\mathcal{D}$ initially processes DB into an encrypted database EDB and sends it to $\mathcal{E}$. $\mathcal{D}$ only keeps a set of cryptographic keys that allows her to later run encrypted searches on $\mathcal{E}$ and decrypt the matching documents returned by $\mathcal{E}$. This setting is known as searchable symmetric encryption (SSE) and has been studied extensively [28, 15, 16, 10, 13, 11, 23]. While most of the research has focused on single-keyword searches (i.e., return all documents containing a given keyword), recently Cash et al. [9] provided the first SSE solution, the OXT protocol, that can support in a practical and private way arbitrary boolean queries on sets of keywords and in very large DBs. The leakage to $\mathcal{E}$, which is formally specified and proven in [9], is in the form of data-access and query patterns, never as direct exposure of plaintext data or searched values.

In this work we are concerned with richer outsourcing scenarios where multiple third parties (clients) access the data at $\mathcal{E}$ but only through queries authorized by the data owner $\mathcal{D}$. For example, consider a hospital outsourcing an (encrypted) database to an external service $\mathcal{E}$ such that clients (doctors, administrators, insurance companies, etc.) can search the database but only via queries authorized according to the hospital's policy and without these clients learning information on non-matching documents. As before, $\mathcal{E}$ should learn as little as possible about data and queries.

In this multi-client scenario (to which we refer as $M C$-SSE), $\mathcal{D}$ provides search tokens to clients based on their queries and according to a given authorization policy. Security considers multiple clients acting maliciously and possibly colluding with each other (trying to gain information beyond what they are authorized for) and a semi-trusted server $\mathcal{E}$ which acts as "honest-but-curious" but does not collude with clients. Extending SSE solutions to the multi-client scenario is straightforward when (a) search tokens are fully determined by the query and (b) the SSE protocol does not return false positives (returning false positives, i.e. documents that do not match a query, is allowed in SSE since the recipient in that case is the owner of the data but not in the multi-client setting where clients are not allowed to learn data they were not authorized for). In such cases, $\mathcal{D}$ would receive the client's query, generate the corresponding SSE search tokens as if $\mathcal{D}$ herself was searching the database, and provide the tokens to the client together with a signature that $\mathcal{E}$ can check before processing 
the search. However, for enabling general boolean queries, the SSE OXT protocol of [9] requires a number of tokens that is not known a-priori (it depends on the searched data, not only on the query) and therefore the above immediate adaptation does not work.

Our first contribution is in extending the OXT protocol from [9] to the MC-SSE setting while preserving its full boolean-query capabilities and performance. In this extension, $\mathcal{D}$ provides the client $\mathcal{C}$ with a set of query-specific trapdoors which the client can then transform into search tokens as required by OXT. The set of trapdoors given to $\mathcal{C}$ is fully determined by the query and independent of the searched data. An additional subtle technical challenge posed by OXT is how to allow $\mathcal{E}$ to verify that the search tokens presented by $\mathcal{C}$ are authorized by $\mathcal{D}$. The simple solution is for $\mathcal{D}$ to sign the trapdoors, however in OXT these trapdoors need to be hidden from $\mathcal{E}$ (otherwise $\mathcal{E}$ can learn information about unauthorized searches) so a simple signature on them cannot be verified by $\mathcal{E}$. Our solution uses a homomorphic signature by $\mathcal{D}$ on the trapdoors that $\mathcal{C}$ can then transform homomorphically into signatures on the search tokens. We show that forging the tokens or their signatures is infeasible even by fully malicious clients.

The resulting MC-OXT protocol preserves the full functional properties of OXT, namely support for arbitrary boolean queries, the same level of privacy (i.e., same leakage profile) with respect to $\mathcal{E}$, and the same remarkable performance. Privacy with respect to clients is near-optimal (see Section 3.1 for why such leakage may be inevitable) with leakage confined only to information on the number of documents matching one of the query terms (typically, the least-frequent term).

Next, we extend the MC-OXT protocol to an even more challenging setting we call Outsourced Symmetric Private Information Retrieval (OSPIR), where on top of the MC$\mathrm{SSE}$ requirements, one asks that client queries be hidden from $\mathcal{D}$ - similarly to the Private Information Retrieval (PIR) primitive. This requirement arises in important outsourcing scenarios. In the medical database example mentioned above, the hospital authorizes doctors or other parties to search the medical database according to certain policy; however, in some cases the actual query values are to be kept secret from the hospital itself (due to privacy, liability and regulatory requirements). Only the minimal information for determining the compliance of a query to the policy should be disclosed to the hospital. For example, the policy may indicate that only conjunctions with a minimal number of terms are allowed or that the query needs to include at least three of a set of attributes, etc. In such a case, there is no need for the hospital to learn the particular values being searched (such as a specific last name or medical condition). In other cases, as in outsourced patent or financial information search, the provider $\mathcal{D}$ may want to enforce that a client $\mathcal{C}$ pays for the type of query it is interested in but $\mathcal{C}$ wants to keep his query hidden from both $\mathcal{D}$ and $\mathcal{E}$. Applications to intelligence scenarios are discussed in [18] (see also [31]).

Thus, we relax the query privacy requirement with respect to $\mathcal{D}$ to allow for minimal information needed for $\mathcal{D}$ to determine policy compliance. Specifically, we consider the case where keywords are formed by pairs of attribute-values. For example, in a relational database, attributes are defined by the database columns (e.g., SSN, name, citizenship, 
etc.), while in an email repository attributes can refer to envelope information such as sender and receivers or to the message body (in which case the values are, say, English words). In this case, a policy defines the class of boolean expressions allowed for a given client and the attributes that may be used as inputs into these expressions. In order to enforce the policy, $\mathcal{D}$ learns the boolean expression and attributes but nothing about the searched values. For policies defined via classes of attributes (e.g. allowing any attribute from the set of attributes name, city, zipcode\}) leakage to $\mathcal{D}$ can be further reduced by revealing the class and not the specific attributes in the query.

Our most advanced result is extending the OXT protocol to the above OSPIR setting. The resultant protocol, OSPIR-OXT, adds some crucial new ingredients to OXT: It uses oblivious PRFs (OPRF) for hiding the query values from $\mathcal{D}$, uses attribute-specific keys for enforcing policy compliance, and uses homomorphic signatures (or the more general abstraction of shared $O P R F s$ ) for query verification by $\mathcal{E}$. A further extension of the protocol accommodates an external policy manager, e.g., a judge in a warrant-based search, who checks policy compliance and allows server $\mathcal{D}$ to enforce the policy without learning the attributes being searched.

Performance-wise our extensions to OXT preserve the protocol's performance in both pre-processing (creating EDB) and search phases. OSPIR-OXT adds to the computational cost by adding a few exponentiations but these are generally inexpensive relative to the I/O cost (especially thanks to common-base exponentiation optimizations). The protocols we provide for MC-SSE and OSPIR models support encrypted search over database containing tens of billions record-keyword pairs, for example a full snapshot of English Wikipedia or a 10-TByte, 100M-record US-census database (see Sections 4.3 and 4.4).

We achieve provable security against adaptive adversarial honest-but-curious server $\mathcal{E}$, against arbitrarily malicious (but non-colluding ${ }^{1}$ with $\mathcal{E}$ ) server $\mathcal{D}$, and against arbitrarily malicious clients. Our security models extend the SSE model $[13,11,9]$ to the more complex settings of MC-SSE and OSPIR. In all cases security is defined in the real-vs-ideal model and is parametrized by a specified leakage function $\mathcal{L}(\mathrm{DB}, \mathbf{q})$. A protocol is said to be secure with leakage profile $\mathcal{L}(\mathrm{DB}, \mathbf{q})$ against adversary $\mathcal{A}$ if the actions of $\mathcal{A}$ on adversarially-chosen input DB and queries set $\mathbf{q}$ can be simulated with access to the leakage information $\mathcal{L}(\mathrm{DB}, \mathbf{q})$ only (and not to DB or $\mathbf{q}$ ). This allows modeling and bounding partial leakage allowed by SSE protocols. It means that even an adversary that has full information about a database, or even chooses it, does not learn anything from the protocol execution other than what can be derived solely from the defined leakage profile.

Related work: Searchable symmetric encryption (SSE) has been extensively studied [28, $15,16,10,13,11,23]$ (see $[13,11]$ for more on related work). Most SSE research focused on single-keyword search, and after several solutions with complexity linear in the database size, Curtmola et al. [13] present the first solution for single-keyword search whose complexity is linear in the number of matching documents. They also improve on previous security

\footnotetext{
${ }^{1}$ See Section 5.2 .
} 
models, in particular by providing an adaptive security definition and a solution in this model.

Extending single-keyword SSE to search by conjunctions of keywords was considered in $[16,7,2]$, but all these schemes had $O(|\mathrm{DB}|)$ search complexity. The first SSE which can handle very large DBs and supports conjunctive queries is the OXT protocol discussed above, given by Cash et al. [9]. The MC-SSE and OSPIR schemes we present are based on this protocol and they preserve its performance and privacy characteristics.

Extension of the two-party client-server model of SSE to the multi-client setting was considered by Curtmola et al, [13], but their model disallowed per-query interaction between the data owner and the client, leading to a relatively inefficient implementation based on broadcast encryption. Multi-client SSE setting which allows such interaction was considered by Chase and Kamara [11] as SSE with "controlled disclosure", and by Kamara and Lauter [22], as "virtual private storage", but both considered only single-keyword queries and did not support query privacy from the data owner. De Cristofaro et al. [12] extended multiclient SSE to the OSPIR setting, which supports query privacy, but only for the case of single-keyword queries. In recent independent work, Pappas et al. [26] provide support for boolean queries in a setting similar to our OSPIR setting (but with honest-but-curious clients).

SSE schemes which support efficient updates of the encrypted database appeared in [29, 23] for single-keyword SSE. The OXT SSE scheme of [9] which supports arbitrary boolean queries, has been extended to the dynamic case in [8], and the same techniques apply to the MC-SSE and OSPIR schemes presented in this paper.

Recently Islam et al. [19] showed that frequency analysis revealed by access control patterns in SSE schemes can be used to predict single-keyword queries. Such attacks, although harder to stage, are possible for conjunctive queries as well, but the general masking and padding countermeasures suggested in [19] are applicable to the MC-OXT and OSPIR-OXT protocols.

In other directions, SSE was extended to the public key setting, allowing any party to encrypt into the database, first for single-keyword search [5, 30, 1, 3, 6, 27], and later for conjunctive queries as well [6], but all these PKSE schemes have $O(|\mathrm{DB}|)$ search complexity. Universally composable SSE was introduced by [24], also with $O(|\mathrm{DB}|)$ search complexity.

Multi-client SSE and OSPIR models are related to the work on multi-client ORAM, e.g. see the recent work of Huang and Goldberg [17], which aims for stronger privacy protection of client's queries from server $\mathcal{E}$, but multi-client ORAM supports DB lookups by (single) indexes instead of (boolean formulas on) keywords, and they can currently support much smaller DB sizes.

Paper organization. We first present our protocols for the case of conjunctive queries: in Section 2 we recall the basic OXT protocol [9], suitably reformulated for our generalizations, in Section 3 we address the multi-client SSE model, and in Section 4 we handle the OSPIR 
model. In Section 4.2 we explain how to extend support for general boolean queries. Security models and claims are presented in Section 5. While the main implementation details and performance analysis is deferred to a companion paper [8], we provide some information on computational cost and performance measurements from our implementation in Section 4.3 and 4.4 , respectively.

\section{SSE and the OXT Protocol}

We first recall the SSE OXT protocol from [9] that forms the basis for our solution to searchable encryption in the more advanced MC and OSPIR models.

SSE protocols and formal setting [9]. Let $\lambda$ be a security parameter. A database $\mathrm{DB}=\left(\operatorname{ind}_{i}, \mathrm{~W}_{i}\right)_{i=1}^{d}$ is a list of identifier and keyword-set pairs, where $\operatorname{ind}_{i} \in\{0,1\}^{\lambda}$ is a document identifier and $\mathrm{W}_{i} \subseteq\{0,1\}^{*}$ is a list of keywords in that document. We set $\mathrm{W}$ to $\bigcup_{i=1}^{d} \mathrm{~W}_{i}$. A query $\psi(\bar{w})$ is specified by a tuple of keywords $\bar{w} \in \mathrm{W}^{*}$ and a boolean formula $\psi$ on $\bar{w}$. We write $\operatorname{DB}(\psi(\bar{w}))$ for the set of identifiers of documents that "satisfy" $\psi(\bar{w})$. Formally, this means that ind in $_{i} \mathrm{DB}(\psi(\bar{w}))$ iff the formula $\psi(\bar{w})$ evaluates to true when we replace each keyword $w_{j}$ with true or false depending on if $w_{j} \in \mathrm{W}_{i}$ or not (in particular $\mathrm{DB}(w)=\left\{\operatorname{ind}_{i}\right.$ s.t. $\left.\left.w \in \mathbf{W}_{i}\right\}\right)$. Below we let $d$ denote the number of records in DB, $m=|\mathrm{W}|$, and $N=\sum_{w \in \mathrm{W}}|\mathrm{DB}(w)|$.

A searchable symmetric encryption (SSE) scheme $\Pi$ consists of an algorithm EDBSetup and a protocol Search fitting the following syntax. EDBSetup takes as input a database DB and a list of document decryption keys RDK, and outputs a secret key $K$ along with an encrypted database EDB. The search protocol proceeds between a client $\mathcal{C}$ and server $\mathcal{E}$, where $\mathcal{C}$ takes as input the secret key $K$ and a query $\psi(\bar{w})$ and $\mathcal{E}$ takes as input EDB. At the end of the protocol $\mathcal{C}$ outputs a set of (ind, rdk) pairs while $\mathcal{E}$ has no output. We say that an SSE scheme is correct if for all DB, RDK and all queries $\psi(\bar{w})$, for $(K$, EDB $) \leftarrow$ EDBSetup $(\mathrm{DB}, \mathrm{RDK})$, after running Search with client input $(K, \phi(\bar{w}))$ and server input EDB, the client outputs $\operatorname{DB}(\phi(\bar{w}))$ and $\operatorname{RDK}[\mathrm{DB}(\phi(\bar{w}))]$ where $\operatorname{RDK}[S]$ denotes $\{\operatorname{RDK}[$ ind $] \mid$ ind $\in S\}$. Correctness can be statistical (allowing a negligible probability of error) or computational (ensured only against computationally bounded attackers - see [9]).

Note (conjunctive vs. Boolean queries). Throughout the paper we present our protocols for the case of conjunctive queries where a query consists of $n$ keywords $\bar{w}=\left(w_{1}, \ldots, w_{n}\right)$ and it returns all documents containing all these keywords. The adaptation to the case of boolean queries is described in Section 4.2.

Note (the array RDK). Our SSE syntax, and the OXT description in Figure 1, includes as input to EDBSetup an array RDK that contains, for each document in DB, a key rdk used to encrypt that document. When a client retrieves the index ind of a document matching its query, it also retrieves the record-decrypting key rdk needed to decrypt that record. This mechanism is not strictly needed in the SSE setting (where rdk could be derived from ind 
using a PRF with a secret key known to $\mathcal{C}$ ) and it is not part of the original OXT in [9], but it is needed in the more advanced models considered later. This extension does not change the functionality and security properties of OXT as analyzed in [9]).

Note (retrieval of matching encrypted records). Our formalism defines the output of the SSE protocol as the set of ind identifiers pointing to the encrypted records matching the query, together with the associated record decryption key. For the sake of generality, we do not model the processing and retrieval of encrypted records. This allows us to decouple the storage and processing of document payloads (which can be done in a variety of ways, with varying types of leakage) from the storage and processing of the metadata, which is the focus of our protocols.

SSE Security. The SSE setting considers security w.r.t. an adversarial server $\mathcal{E}$, hence security is parametrized via a leakage function capturing information learned by $\mathcal{E}$ from the interaction with $\mathcal{C}$. See Section 5 .

The TSet Datastructure for Inverted Index. The SSE protocol OXT, on which our MC-SSE and OSPIR-SSE schemes are based, uses a datastructure TSet which abstracts an inverted index (a list) in a way which minimizes leakage of information to the server $\mathcal{E}$ on which this index is stored. We adopt the API for this datastructure given by [9]. Specifically, the abstract TSetSetup operation receives a collection $\mathbf{T}$ of lists $\mathbf{T}[w]$ for each $w \in \mathbf{W}$ and builds the TSet data structure out of these lists; it returns TSet and a key $K_{T}$. Then, for any $w$, procedure TSetGetTag $\left(K_{T}, w\right)$ (typically a PRF) generates a search handle, denoted stag, which allows for retrieval of $\mathbf{T}[w]$ from TSet, via procedure TSetRetrieve(TSet, stag). By convention we define $\mathbf{T}[w]$ as an empty list for every bitstring $w \notin \mathbf{W}$. The elements in the $\mathbf{T}$ lists are called tuples, hence the name "TSet" which stands for a "tuple set", and their exact contents are defined by the OXT protocol. A TSet implementation as defined in [9] must provide privacy, in that the TSet datastructure does not reveal anything about the tuple lits in $\mathbf{T}$ except their total size $\sum_{w}|\mathbf{T}[w]|$; and correctness, in that TSetRetrieve(TSet, stag) returns $\mathbf{T}[w]$ for stag $=\operatorname{TSetGet} \operatorname{Tag}\left(K_{T}, w\right)$. However, here we extend the above correctness property, because unlike the two-party SSE settings of [9], in the MC-SSE or OSPIR-SSE settings we must require TSet implementation to have no false positives in addition to no false negatives. We formally specify this extended notion of TSet correctness in Section 5.1.

\subsection{The OXT Protocol}

The OXT protocol [9] is presented in Figure 1; see [9] for full design rationale and analysis. Here we provide a high level description as needed for the extensions to this protocol we introduce in the following sections.

The basis of OXT is the following simple search algorithm over unencrypted databases. The algorithm uses two types of data structures. First, for every keyword $w$ there is an inverted index (a list) $I_{w}$ pointing to the indices ind of all documents that contain $w$. Then, for every document ind there is a list $L_{\text {ind }}$ of all keywords contained in document ind. To 
EDBSetup(DB, RDK)

Key Generation. $\mathcal{D}$ picks key $K_{S}$ for PRF $F_{\tau}$ and keys $K_{T}, K_{X}, K_{I}$ for PRF $F_{p} . F_{\tau}$ and $F_{p}$ are PRF's which output strings in respectively $\{0,1\}^{\tau}$ and $Z_{p}^{*}$, and $\tau$ is a security parameter.

- Initialize XSet to an empty set, and initialize $\mathbf{T}$ to an empty array indexed by keywords from $\mathbf{W}$.

- For each $w \in \mathbf{W}$, build the tuple list $\mathbf{t}$ and insert elements into set XSet as follows:

- Initialize $\mathbf{t}$ to be an empty list.

- Set strap $\leftarrow F_{\tau}\left(K_{S}, w\right)$ and $\left(K_{z}, K_{e}\right) \leftarrow\left(F_{\tau}(\right.$ strap, 1$), F_{\tau}($ strap, 2$\left.)\right)$.

- Initialize $c \leftarrow 0$; then for all ind in $\mathrm{DB}(w)$ in random order:

$*$ Set $r d k \leftarrow \operatorname{RDK}($ ind $), \mathrm{e} \leftarrow \operatorname{Enc}\left(K_{e}\right.$, (ind $\left.\left.\mid r d k\right)\right)$, xind $\leftarrow F_{p}\left(K_{I}\right.$, ind $)$.

$*$ Set $c \leftarrow c+1, z_{c} \leftarrow F_{p}\left(K_{z}, c\right), y \leftarrow$ xind $\cdot z_{c}^{-1}$. Append (e, $\left.y\right)$ to t.

* Set $x \operatorname{tag} \leftarrow g^{F_{p}\left(K_{X}, w\right) \cdot x i n d}$ and add xtag to XSet.

$-\mathbf{T}[w] \leftarrow \mathbf{t}$.

- Create $\left(\mathrm{TSet}, K_{T}\right) \leftarrow \mathrm{TSetSetup}(\mathbf{T})$, and output key $K=\left(K_{S}, K_{X}, K_{T}, K_{I}\right)$ and EDB $=(\mathrm{TSet}, \mathrm{XSet})$.

Search protocol

Client $\mathcal{C}$ on input $K$ defined as above and a conjunctive query $\bar{w}=\left(w_{1}, \ldots, w_{n}\right)$ :

- Sets stag $\leftarrow \operatorname{TSetGetTag}\left(K_{T}, w_{1}\right)$; strap $\leftarrow F_{\tau}\left(K_{S}, w_{1}\right),\left(K_{z}, K_{e}\right) \leftarrow\left(F_{\tau}(\operatorname{strap}, 1), F_{\tau}(\operatorname{strap}, 2)\right)$.

- Sends to the server $\mathcal{E}$ the message $(\operatorname{stag}, x \operatorname{token}[1], x \operatorname{token}[2], \ldots)$ where $x \operatorname{token}[\cdot]$ are defined as:

- For $c=1,2, \ldots$, until $\mathcal{E}$ sends stop:

* Set $z_{c} \leftarrow F_{p}\left(K_{z}, c\right)$ and set xtoken $[c, i] \leftarrow g^{F_{p}\left(K_{X}, w_{i}\right) \cdot z_{c}}$ for $i=2, \ldots, n$.

* Set xtoken $[c] \leftarrow($ xtoken $[c, 2], \ldots, x \operatorname{xtoken}[c, n])$.

Server $\mathcal{E}$ on input $\mathrm{EDB}=(\mathrm{TSet}, \mathrm{XSet})$ responds as follows:

- Retrieve $\mathbf{t} \leftarrow$ TSetRetrieve(TSet, stag) from TSet.

- For $c=1, \ldots,|\mathbf{t}|$ do:

- Retrieve (e, $y$ ) from the $c$-th tuple in $\mathbf{t}$

- If $\forall i=2, \ldots, n:$ xtoken $[c, i]^{y} \in \mathrm{XSet}$ then send e to client $\mathcal{C}$.

- When last tuple in $\mathbf{t}$ is reached, sends stop to $\mathcal{C}$ and halt.

For each received e client $\mathcal{C}$ computes (ind $\mid r d k) \leftarrow \operatorname{Dec}\left(K_{e}\right.$, e) and outputs (ind, rdk).

Figure 1: OXT: Oblivious Cross-Tags SSE Scheme

search for a conjunction $\bar{w}=\left(w_{1}, \ldots, w_{n}\right)$, the client chooses the estimated least frequent 
keyword ${ }^{2}$ in $\bar{w}$, say $w_{1}$, and checks for each ind $\in I_{w_{1}}$ whether $w_{i} \in L_{\text {ind }}, i=2, \ldots, n$. If this holds for all $2 \leq i \leq n$ then ind is added to the result set. As a performance optimization, instead of maintaining a list $L_{\text {ind }}$ for each ind, one can fix a hash function $f$ and keep a data structure representing the set $X=\{f(w$, ind $): w \in \mathrm{W}$, ind $\in \mathrm{DB}(w)\}$. Thus, the check $w \in L_{\text {ind }}$ can be replaced with the check $f(w$, ind $) \in X$. Protocol OXT adapts this algorithm to the encrypted setting as follows (we start with the description of a simplified version, corresponding to protocol BXT in [9], and then move to the more specific details of OXT).

For each keyword $w \in \mathrm{W}$ an inverted index $\operatorname{TSet}(w)$ (corresponding to $I_{w}$ above) is built pointing to all the ind values of documents in $\operatorname{DB}(w)$. Each TSet $(w)$ is identified by a string $\operatorname{stag}(w)$, and ind values in TSet $(w)$ are encrypted under a secret key $K_{e}$. Both $\operatorname{stag}(w)$ and $K_{e}$ are computed as a PRF applied to $w$ with secret keys known to $\mathcal{C}$ only. In addition, a data structure called XSet is built as an "encrypted equivalent" of the above set $X$ as follows. First, for each $w \in \mathrm{W}$, a value $\operatorname{xtrap}(w)=F\left(K_{X}, w\right)$ is computed where $K_{X}$ is a secret PRF key then for each ind $\in \mathrm{DB}(w)$ a value $\times \operatorname{tag}=f(\operatorname{xtrap}(w)$, ind $)$ is added to XSet where $f$ is an unpredictable function of its inputs (e.g., $f$ can be a PRF used with $x \operatorname{trap}(w)$ as the key and ind as input). The output EDB from the EDBSetup phase includes TSet $=\{\operatorname{TSet}(w)\}_{w \in W}$ and the set XSet. In the Search protocol for a conjunction $\left(w_{1}, \ldots, w_{n}\right)$, the client $\mathcal{C}$ chooses the conjunction's s-term (i.e., the estimated least frequent keyword in the conjunction, which we assume to be $\left.w_{1}\right)$, computes $\operatorname{stag}\left(w_{1}\right)$ and $K_{e}$ using $\mathcal{C}$ 's secret keys and computes $\operatorname{xtrap}_{i}=F\left(K_{X}, w_{i}\right)$ for each $i=2, \ldots, n$. It then sends $\left(K_{e}, \operatorname{stag}_{1}, \operatorname{xtrap}_{2}, \ldots, \operatorname{xtrap}_{n}\right)$ to the server $\mathcal{E}$. $\mathcal{E}$ uses stag to retrieve $\operatorname{TSet}\left(w_{1}\right)$, uses $K_{e}$ to decrypt the ind values in $\operatorname{TSet}\left(w_{1}\right)$, and sends back to $\mathcal{C}$ those ind for which $f\left(\operatorname{xtrap}_{i}\right.$, ind $) \in \mathrm{XSet}$ for all $i=2, \ldots, n$.

Next, we note that using the protocol described above leads to significant leakage in that the $x$ trap value allows $\mathcal{E}$ to check whether $\times$ tag $=f($ xtrap, ind $) \in$ XSet for each value ind ever seen by $\mathcal{E}$, revealing correlation statistics between each s-term and each x-term ever queried by $\mathcal{C}$. This motivates the main mechanism in OXT, i.e. the instantiation of the function $f$ via a two-party computation in which $\mathcal{E}$ inputs an encrypted value ind, $\mathcal{C}$ inputs xtrap and the ind-decryption key, and $\mathcal{E}$ gets the value of xtag $=f$ (xtrap, ind) without learning either the xtrap or ind values themselves. For this OXT uses a blinded DH computation over a group $G$ (with generator $g$ of prime order $p$ ). However, to avoid the need for interaction between $\mathcal{E}$ and $\mathcal{C}$ in the Search phase, the blinding factors are pre-computed and stored as part of the tuples in the TSet lists. Specifically, indexes ind are replaced in the computation of $f$ with dedicated per-record values xind $\in Z_{p}^{*}$ (computed as $F_{p}\left(K_{I}\right.$, ind) where $F_{p}$ is a PRF with range $\left.Z_{p}^{*}\right)$, xtrap $(w)$ 's are implemented as $g^{F_{p}\left(K_{X}, w\right)}\left(K_{I}\right.$ and $K_{X}$ are secret keys kept by $\mathcal{C})$, and $x \operatorname{tag}$ is re-defined as $(\operatorname{xtrap}(w))^{\text {xind }}$. The blinding factor in the underlying two-party computation is pre-computed during EDBSetup and stored in the TSet. Namely, each tuple corresponding to a keyword $w$ and document index ind contains a blinded value $y_{c}=$ xind $\cdot z_{c}^{-1}$ for xind $=F_{p}\left(F_{I}\right.$, ind) where $z_{c}$ is an element in $Z_{p}^{*}$ derived (via a PRF) from $w$ and a tuple counter $c$ (this counter, incremented for each tuple in the tuple list associated

\footnotetext{
${ }^{2}$ The estimated least frequent keyword is called the conjunction's s-term; the other terms in a conjunction are called $x$-terms.
} 
with $w$, ensures independence of each blinding value $z_{c}$ ).

During search, the server $\mathcal{E}$ needs to compute the xtag values $g^{F_{p}\left(K_{X}, w_{i}\right) \cdot x \text { ind }}$ for each xind in $\operatorname{TSet}\left(w_{1}\right)$ and then test these for membership in XSet. To enable this the client sends, for the $c$-th tuple in $\mathbf{t}$, an $n$-long array xtoken $[c]$ defined by xtoken $[c, i]:=g^{F_{p}\left(K_{X}, w_{i}\right) \cdot z_{c}}$, $i=1, \ldots, n$, where $z_{c}$ is the precomputed blinding derived by $\mathcal{C}$ from $w$ (via a PRF) and the tuple counter $c$. $\mathcal{E}$ then performs the T-set search to get the results for $w_{1}$, and for each $c$ it filters the $c$-th result by testing if xtoken $[c, i]^{y_{c}} \in \mathrm{XSet}$ for all $i=2, \ldots, n$. This protocol is correct because xtoken $[c, i]^{y_{c}}=g^{F_{p}\left(K_{X}, w_{i}\right) \cdot z_{c} \cdot \text { xind } \cdot z_{c}^{-1}}=g^{F_{p}\left(K_{X}, w_{i}\right) \cdot \text { xind }}$, meaning that the server correctly recomputes the pseudorandom values in the XSet. Putting these ideas together results in the OXT protocol of Figure 1. Note that $\mathcal{C}$ sends the xtoken arrays (each holding several values of the form $g^{F_{p}\left(K_{X}, w_{i}\right) \cdot z_{c}}$ ) until instructed to stop by $\mathcal{E}$. There is no other communication from server to client (alternatively, server can send the number of elements in TSet $(w)$ to the client who will respond with such number of xtoken arrays).

Note. The OXT protocol in Figure 1 derives keys $K_{e}, K_{z}$ slightly differently than in the OXT description of [9]. The modified key derivation is closer to what we need for MC-OXT and OSPIR-OXT protocols presented in the following sections, without affecting the functionality or security of OXT.

\section{Multi-Client SSE}

We present an extension of the OXT protocol for the multi-client SSE (MC-SSE) setting described in the introduction and in more detail below. The extension preserves the functionality of OXT, supporting any boolean query, and superb performance, while securely serving multiple clients, all of which can behave maliciously.

Multi-Client SSE Setting. In MC-SSE there is the owner $\mathcal{D}$ of the plaintext database $\mathrm{DB}$, an external server $\mathcal{E}$ that holds the encrypted database EDB, and clients that receive tokens from $\mathcal{D}$ in order to perform search queries at $\mathcal{E}$. In other words, $\mathcal{D}$ is outsourcing her search service to a third party but requires clients to first obtain search tokens from her. Her goal is to ensure service to clients via $\mathcal{E}$ while leaking as little as possible information to $\mathcal{E}$ about the plaintext data and queries, and preventing clients from running any other DB queries than those for which $\mathcal{D}$ issued them a token. This is a natural outsourcing setting of increasing value in cloud-based platforms, and it was described by Chase and Kamara [11] as an SSE with controlled disclosure.

Formally, the MC-SSE setting changes the syntax of an SSE scheme by including an additional algorithm GenToken which on input the secret key $K$, generated by the data owner $\mathcal{D}$ in the EDBSetup procedure, and a boolean query $\psi(\bar{w})$, submitted by client $\mathcal{C}$, generates a search-enabling value token. Then, procedure Search is executed by server $\mathcal{E}$ on input EDB, but instead of the client $\mathcal{C}$ running on input $K$ and query $\psi(\bar{w})$ (as in SSE), $\mathcal{C}$

runs on input consisting only of the search token token. Correctness is defined similarly to 
the SSE case, namely, assuring (except for negligible error probability) that $\mathcal{C}$ 's output sets $\operatorname{DB}(\psi(\bar{w}))$ and $\operatorname{RDK}[\operatorname{DB}(\psi(\bar{w}))]$. Security is treated in Section 5 .

\subsection{The MC-OXT Protocol}

We describe the changes to OXT from Figure 1 needed to support boolean queries in the MC-SSE setting. As before, the protocol is described for conjunctions with the adaptation to boolean queries described in Section 4.2.

EDBSetup(DB, RDK). This pre-processing phase is identical to the one in OXT except for the addition of a key $K_{M}$ shared between $\mathcal{D}$ and $\mathcal{E}$. The output from this phase is $K=\left(K_{S}, K_{X}, K_{T}, K_{M}\right)$ kept by $\mathcal{D}$ and $\mathrm{EDB}=\left(K_{M}\right.$, TSet, XSet $)$ stored at $\mathcal{E}$.

$\operatorname{Gen} \operatorname{Token}(K, \bar{w})$. This is the new MC-specific phase in which $\mathcal{D}$, using key $K$, authorizes $\mathcal{C}$ for a conjunction $\bar{w}=w_{1}, \ldots, w_{n}$ and provides $\mathcal{C}$ with the necessary tokens to enable the search at $\mathcal{E}$. We assume $w_{1}$ to be the s-term (and chosen by $\mathcal{D}$ who has knowledge of term frequencies). Specifically, $\mathcal{D}$ performs the following operations. She sets stag $\leftarrow$ TSetGetTag $\left(K_{T}, w_{1}\right)$ and strap $\leftarrow F_{\tau}\left(K_{S}, w_{1}\right)$, same as $\mathcal{C}$ does in OXT. Then, for $i=2, \ldots, n$, she picks $\rho_{i} \leftarrow Z_{p}^{*}$ and sets bxtrap $i \leftarrow g^{F_{p}\left(K_{X}, w_{i}\right) \cdot \rho_{i}}$. Finally, she sets env $\leftarrow \operatorname{AuthEnc}\left(K_{M}\right.$, $\left(\right.$ stag $\left.\left., \rho_{2}, \ldots, \rho_{n}\right)\right)$ and outputs token $=\left(\right.$ env, strap, bxtrap $_{2}, \ldots$, bxtrap $\left._{n}\right)($ which $\mathcal{C}$ uses in the search phase).

To see how this enables search as in OXT, first note that with $\operatorname{xtrap}_{i}=g^{F_{p}\left(K_{X}, w_{i}\right)}$, $i=2, \ldots, n$, the client can produce the values $g^{F_{p}\left(K_{X}, w_{i}\right) \cdot z_{c}}$ needed in the Search phase of OXT. Hence, $\mathcal{D}$ could just provide the $\operatorname{xtrap}_{i}$ values to $\mathcal{C}$. However, $\mathcal{D}$ needs to be able to sign (or MAC) these values so that $\mathcal{E}$ can check that $\mathcal{C}$ is authorized to this query and, e.g., did not truncate a conjunction or mix parts from different queries. Signing the plain xtrap values does not work since these values must not be seen by $\mathcal{E}$ (it would allow $\mathcal{E}$ to learn information from unauthorized searches). The solution is to provide $\mathcal{C}$ with a homomorphic signature that $\mathcal{C}$ can convert into individual signatures for all tokens sent to $\mathcal{E}$. This is accomplished as follows: $\mathcal{D}$ picks one-time blinding factors $\rho_{2}, \ldots, \rho_{n}$ and provides $\mathcal{C}$ with blinded (or MAC'ed) xtrap values bxtrap $_{i}=\operatorname{xtrap}_{i}^{\rho_{i}}$ for $j=2, \ldots, n$, while providing the blinding factors $\left(\rho_{2}, \ldots, \rho_{n}\right)$ to $\mathcal{E}$ in an encrypted and authenticated envelope env. To produce $g^{F_{p}\left(K_{X}, w_{i}\right) \cdot z_{c}}$ during Search, $\mathcal{C}$ will compute bxtrap $_{i}^{z_{c}}$ and $\mathcal{E}$ will raise this value to $1 / \rho_{i}$. Security relies on the fact that if $\mathcal{C}$ provides $\mathcal{E}$ with values other than those given by $\mathcal{D}$, then when raised to $1 / \rho_{i}$ by $\mathcal{E}$ (where $\rho_{i}$ is random and unknown to $\mathcal{C}$ ) the resulting values will not correspond to elements of XSet except with negligible probability.

Search Protocol. The Search protocol reflects the above changes: On token $=\left(\right.$ env, strap, bxtrap ${ }_{2}, \ldots$, bxtrap $\left._{n}\right), \mathcal{C}$ computes $\left(K_{z}, K_{e}\right)$ from strap as in OXT. Then, it sends to $\mathcal{E}$ the value env as well as the sequence bxtoken[1], bxtoken[2],... which contains the same values as xtoken[1], xtoken[2], .. in OXT up to the blinding exponents $\rho_{i}$. Specifically, the values bxtoken $[c, i]$, for $i=2, \ldots, n$, are computed by $\mathcal{C}$ as $\left(\text { bxtrap }_{i}\right)^{z_{c}}$. On the receiving side, $\mathcal{E}$ verifies the authenticity of env and decrypts it to find stag, which it uses to retrieve TSet $\left(w_{1}\right)$, 
and $\rho_{2}, \ldots, \rho_{n}$. The only change from OXT is that the operation xtoken $[c, i]^{y}$ is replaced with bxtoken $[c, i]^{y / \rho_{i}}$.

Note (masking the size of TSet $\left(w_{1}\right)$ ). MC-OXT leaks to $\mathcal{C}$ the size of $\operatorname{TSet}\left(w_{1}\right)=\mathrm{DB}\left(w_{1}\right)$ given by the number of bxtoken vectors requested by $\mathcal{E}$. Note that the exact size of this set can easily be masked by $\mathcal{E}$ requesting more bxtoken values from $\mathcal{C}$ than needed. We observe that some form of leakage on the frequency of the least frequent term in the conjunction appears to be inherent even for plaintext search algorithms. Indeed, it is likely (though there seem to be no proven lower bounds) that running time will be noticeably different for conjunctions with all terms being infrequent from the case that all terms are very frequent, except if short searches are artificially padded to full database size (or if conjunctions are pre-computed). Two considerations in masking the size of TSet $\left(w_{1}\right)$ are that (i) the masked size should be chosen as a step function of $\left|\operatorname{TSet}\left(w_{1}\right)\right|$ (and not, say, a fixed linear function); (ii) During search, $\mathcal{E}$ should return to $\mathcal{C}$ all results matching a query only after $\mathcal{E}$ received all the bxtoken vectors from $\mathcal{C}$; indeed, sending the results as soon as they are found to match the query would leak information on $|\operatorname{TSet}(w)|$ to $\mathcal{C}$ (since no ind's will be returned after $\mathcal{C}$ sends $|\operatorname{TSet}(w)|$ tokens). Alternatively, to avoid delaying the sending of results from $\mathcal{E}$ to $\mathcal{C}$, $\mathcal{D}$ can randomly spread the counters $c$ in each TSet $(w)$ during the EDBSetup procedure, so that counters $c$ range between 1 and $|\operatorname{Mask}(\operatorname{TSet}(w))|$ rather than between 1 and $|\operatorname{TSet}(w)|$ (note that the number of tuples in $\operatorname{TSet}(w)$ is not increased by this approach, only the counters in these tuples are more sparsely allocated).

\section{Outsourced Symmetric PIR}

The OSPIR (for Outsourced Symmetric PIR) setting augments the MC-SSE setting with an additional requirement: The database owner $\mathcal{D}$ should learn as little as possible about queries performed by clients while still being able to verify the compliance of these queries to her policy. Here we extend MC-OXT to this setting by augmenting the token generation component GenToken to support "blinding" by the client of query requests sent to $\mathcal{D}$, and adding a mechanism for $\mathcal{D}$ to enforce policy-compliance of the query when only having access to their blinded versions. OSPIR-OXT supports attribute-based policies where $\mathcal{D}$ learns information about query attributes but nothing about values (e.g., $\mathcal{D}$ may learn that a query includes a last-name, a zipcode and two words from a text field but nothing about the actual queried keywords). The security model adds $\mathcal{D}$ as a new adversarial entity trying to learn $\mathcal{C}$ 's hidden query values. Refer to the introduction for more discussion about the OSPIR setting and attribute-based policies.

OSPIR SSE Syntax. An OSPIR-SSE scheme replaces the GenToken procedure which in MC-SSE is executed by the data owner $\mathcal{D}$ on the cleartext client's query $\bar{w}$, with a twoparty protocol between $\mathcal{C}$ and $\mathcal{D}$ that allows $\mathcal{C}$ to compute a search-enabling token without $\mathcal{D}$ learning $\bar{w}$. In addition, $\mathcal{D}$ should be able to enforce an attribute-based query-authorization 
policy on these queries. For this, we assume that keyword set $\mathrm{W}$ is partitioned into $m$ attributes, and let $I(w)$ denote the attribute of keyword $w .^{3}$ An attribute-based policy is represented by a set of attribute-sequences $\mathbf{P}$ s.t. a conjunctive query $\bar{w}=\left(w_{1}, \ldots, w_{n}\right)$ is allowed by policy $\mathbf{P}$ if and only if the sequence of attributes $\operatorname{av}(\bar{w})=\left(I\left(w_{1}\right), \ldots, I\left(w_{n}\right)\right)$ corresponding to this query is an element in set P. Using this notation, the goal of the GenToken protocol is to let $\mathcal{C}$ compute token corresponding to its query $\bar{w}$ only if $\operatorname{av}(\bar{w}) \in \mathrm{P}$. Reflecting these goals, an OSPIR-SSE scheme is a tuple $\Sigma=$ (EDBSetup, GenToken, Search) where EDBSetup is an algorithm run by $\mathcal{D}$ on inputs (DB, RDK) with outputs (EDB, $K$ ), GenToken is a protocol run by $\mathcal{C}$ on input $\bar{w}$ and by $\mathcal{D}$ on input $(\mathrm{P}, K)$, with $\mathcal{C}$ outputting token or $\perp$ and $\mathcal{D}$ outputting av, and Search is a protocol run by $\mathcal{C}$ on input token and by $\mathcal{E}$ on input EDB, with $\mathcal{C}$ outputting a set of ind's matching his query and the corresponding set of rdk's.

\subsection{The OSPIR-OXT Protocol}

\section{GenToken protocol}

- Client $\mathcal{C}$, on input $\bar{w}=\left(w_{1}, \ldots, w_{n}\right)$ where $w_{1}$ is chosen as s-term:

- Compute $\left(a_{s}, r_{s}\right) \leftarrow$ OPRF. $\mathcal{C}_{1}\left(w_{1}\right)$, and $\left(a_{i}, r_{i}\right) \leftarrow$ S-OPRF. $\mathcal{C}_{1}\left(w_{i}\right)$ for each $i=1, \ldots, n$.

- Send $\left(a_{s}, a_{1}, \ldots, a_{n}\right)$ and av $=\left(I\left(w_{1}\right), \ldots, I\left(w_{n}\right)\right)$ to $\mathcal{D}$.

- Data owner $\mathcal{D}$, on input policy $\mathrm{P}$ and master key $K=\left(K_{S}, K_{X}, K_{T}, K_{I}, K_{P}, K_{M}\right)$ :

- Abort if av is not in policy set P. Otherwise set av as D's local output.

- Compute $b_{s} \leftarrow$ OPRF.D $\left(K_{S}, a_{s}\right)$.

- Compute $\left(b_{1}, \rho_{1}\right) \leftarrow$ S-OPRF.D $\left(K_{T}, I_{1}, a_{1}\right)$, and $\left(b_{i}, \rho_{i}\right) \leftarrow$ S-OPRF.D $\left(K_{X}, I_{i}, a_{i}\right)$ for $i=2, . ., n$.

- Set env $\leftarrow \operatorname{AuthEnc}\left(K_{M},\left(\rho_{1}, \rho_{2}, \ldots, \rho_{n}\right)\right)$ and send (env, $\left.b_{s}, b_{1}, \ldots, b_{n}\right)$ to $\mathcal{C}$.

- $\mathcal{C}$ outputs token $=\left(\right.$ env, strap, bstag, bxtrap $2, \ldots$, bxtrap $\left._{n}\right)$ where strap $\leftarrow$ OPRF $\mathcal{C}_{2}\left(b_{s}, r_{s}\right)$, bstag $\leftarrow$ S-OPRF. $\mathcal{C}_{2}\left(b_{1}, r_{1}\right)$, and bxtrap $_{i} \leftarrow$ S-OPRF. $\mathcal{C}_{2}\left(b_{i}, r_{i}\right)$ for $i=2, \ldots, n$.

\section{Figure 2: Token Generation In OSPIR-OXT}

The OSPIR-OXT protocol addresses the above OSPIR setting by enhancing MC-OXT with query-hiding techniques that allow $\mathcal{D}$ to authorize queries without learning the queried values. Most changes with respect to MC-OXT are in the GenToken protocol. EDBSetup remains mostly unchanged except for the implementation of the PRFs, and Search is essentially unmodified.

We introduce the two main tools used in the design of GenToken. First, instead of the

\footnotetext{
${ }^{3}$ We assume that the string representing $w$ has its attribute encoded into it, i.e. $w=(i, v a l)$ for $i=I(w)$, so that Ryan as a first name is distinguished from Ryan as a last name.
} 
use of regular PRFs for stag and xtag computations in OXT and MC-OXT, the OSPIROXT protocol uses an an "oblivious PRF" (OPRF) computation between $\mathcal{C}$ and $\mathcal{D}$. A PRF $F(K, w)$ is called oblivious [25] if there is a two-party protocol in which $\mathcal{C}$ inputs $w, \mathcal{D}$ inputs $K, \mathcal{C}$ learns the value of $F(K, w)$ and $\mathcal{D}$ learns nothing. A simple example is the Hashed DH OPRF which we use in our implementation of the OSPIR-OXT protocol, defined as $F(K, x)=H(x)^{K}$ where $H$ is a hash function onto $G \backslash\{1\}$ where $G$ is a group of prime order $p$, and $K$ is chosen at random in $Z_{p}^{*}$. In this case, the OPRF protocol consists of $\mathcal{C}$ sending $a=H(x)^{r}$ for random $r$ in $Z_{p}^{*}$, $\mathcal{D}$ sending back $b=a^{K}$ and $\mathcal{C}$ computing $H(x)^{K}$ as $b^{1 / r}$. The second tool used in GenToken is needed to enforce an attribute-based policy and guarantee that only queries on authorized attributes can generate valid tokens for search at $\mathcal{E}$. To enforce such policies we have $\mathcal{D}$ use a different key for each possible attribute; for example, when a stag (or xtag) is requested for attribute 'zipcode' the key that $\mathcal{D}$ inputs into the OPRF computation is different than the key used for attribute 'name' or attribute 'text'. The point is that if $\mathcal{C}$ claims to be querying zipcode but actually enters the keyword "Michael" into the OPRF computation, the output for $\mathcal{C}$ will be the $\operatorname{tag} F\left(K_{z i p}\right.$, "Michael"), where $K_{z i p}$ is a zipcode-specific key, which will match no tag stored at $\mathcal{E}$.

To obtain OSPIR-OXT we combine the above two tools with an authorization mechanism similar to the one used in MC-OXT via a homomorphic signature (using the $\rho_{i}$ exponents) for binding together the $n$ tokens corresponding to an $n$-term conjunctive query in a way that $\mathcal{E}$ can verify. We describe the changes to MC-OXT (defined via Figure 1 and the modifications in Section 3) required by OSPIR-OXT. We first replace the PRF $F_{p}$ used in computing xtrap and xtag values with a $\mathrm{PRF} F_{G}$ which maps $w$ directly onto the group $G$ generated by $g$, i.e. we set xtrap as $F_{G}\left(K_{X}, w\right)$ instead of $g^{F_{p}\left(K_{X}, w\right)}$, hence xtag $=(\text { xtrap })^{\text {xind }}$ will now be computed as $F_{G}\left(K_{X}, w\right)^{\text {xind }}$ instead of $g^{F_{p}\left(K_{X}, w\right) \cdot x i n d}$. We similarly replace the PRF $F_{\tau}$ used in computing the strap value with the PRF $F_{G}$, i.e. we set strap as $F_{G}\left(K_{S}, w\right)$ instead of $F_{\tau}\left(K_{S}, w\right)$. (Since we use strap as a key to $F_{\tau}$ in deriving $\left(K_{z}, K_{e}\right)$, we assume that a PRF $F_{\tau}$ key can be extracted from a random group element.)

We also make a specific assumption on the implementation of the function TSetGetTag used to derive $\operatorname{stag}(w)$ value, i.e., the handle pointing to the set TSet $(w)$ which is computed as TSetGetTag $\left(K_{T}, w\right)$. First, we assume that TSetGetTag is implemented using PRF $F_{G}$. Second, to enable enforcement of attribute-based policies we assume that the key $K_{T}$ in TSetGetTag is formed by an array of $F_{G}$ keys $K_{T}=\left(K_{T}[1], \ldots, K_{T}[m]\right)$, where $K_{T}[i]$ is the key to be used only for keywords with attribute $I(w)=i$. For notational convenience we define a $\operatorname{PRF} F_{G}^{m}$ s.t. $F_{G}^{m}\left(K_{T}, w\right)=F_{G}\left(K_{T}[I(w)], w\right)$, and we set $\operatorname{stag}(w)=$ $\operatorname{TSetGetTag}\left(K_{T}, w\right)$ to $F_{G}^{m}\left(K_{T}, w\right)$. Since we explicitly handle the keys used in the TSetGetTag implementation we also need to modify the TSet API: We will initialize TSet as TSet $\leftarrow$ TSetSetup $^{\prime}(\mathbf{T})$, where $\mathbf{T}$ indexes the tuple lists $\mathbf{t}(w)$ not by the keywords $w$ but by the corresponding $\operatorname{stag}(w)$ values. (This API change does not affect existing TSet implementations [9] because they internally use $\operatorname{stag}(w)=\operatorname{TSetGet\operatorname {Tag}}\left(K_{T}, w\right)$ to store the $\mathbf{t}(w)$ list.) The PRF we use in the computation of xtag's will be similarly attribute-partitioned. Namely, $K_{X}$ is also an array of $m$ independent $F_{G}$ keys $K_{X}=\left(K_{X}[1], \ldots, K_{X}[m]\right)$, the xtrap value 
for keyword $w$ is defined as $F_{G}^{m}\left(K_{X}, w\right)$, and the xtag corresponding to keyword $w$ and index xind is set to $\left(F_{G}^{m}\left(K_{X}, w\right)\right)^{\text {xind }}$.

In OSPIR-OXT, there are two two-party protocols involved in the computation of $F_{G}$. In the first case, the protocol implements an OPRF computation in which $\mathcal{C}$ enters an input $w$, $\mathcal{D}$ enters a key $K_{S}$, and the output is $F_{G}\left(K_{S}, w\right)$ for $\mathcal{C}$ and $\perp$ for $\mathcal{D}$. In the second case, $F_{G}^{m}$ is computed via a protocol, that we call a shared OPRF (S-OPRF), in which $\mathcal{C}$ inputs $w$ and $i=I(w)$, and $\mathcal{D}$ enters a key $K$ and additional input $\rho \in Z_{p}^{*}$; the output learned by $\mathcal{D}$ is $i$, and the output learned by $\mathcal{C}$ is $\left(F_{G}^{m}(K, x)\right)^{\rho}=\left(F_{G}(K[i], x)\right)^{\rho}$. Note that the pair of outputs $\left(\left(F_{G}^{m}(K, x)\right)^{\rho}, \rho\right)$ can be seen as a secret sharing of $F_{G}^{m}(K, x)$, hence the name shared-OPRF. OSPIR-OXT uses the OPRF protocol to let $\mathcal{C}$ learn the strap value corresponding to the $w_{1}$ s-term, i.e. strap $=F_{G}\left(K_{S}, w_{1}\right)$, without $\mathcal{D}$ learning $w_{1}$. The S-OPRF protocol is used to let $\mathcal{C}$ compute a blinded stag bstag $=\left[F_{G}^{m}\left(K_{T}, w_{1}\right)\right]^{\rho_{1}}$ and the blinded xtraps bxtrap $_{i}=\left[F_{G}^{m}(\right.$ $\left.\left.K_{I}, w_{i}\right)\right]^{\rho_{i}}$, for $i=2, \ldots, n$. The functionality of the blinding $\rho_{i}$ is the same as in the case of MC-OXT, namely, as a form of homomorphic signature binding and authorizing stag and xtrap's that $\mathcal{E}$ can verify. As in MC-OXT, $\mathcal{E}$ will receive the corresponding (de)blinding factors $\rho_{1}, \ldots, \rho_{n}$ in the authenticated envelope env.

To simplify the description of OSPIR-OXT, we assume that both OPRF and S-OPRF protocols take a single round of interaction between $\mathcal{C}$ and $\mathcal{D}$, as is indeed the case for several efficient OPRF's of interest [14, 20], including the Hashed Diffie-Hellman OPRF [21] used in our implementation below. We denote $\mathcal{C}$ 's initial computation in the OPRF protocol as $(a, r) \leftarrow$ OPRF. $\mathcal{C}_{1}(x)$ ( $a$ is the value sent to $\mathcal{D}$ and $r$ is randomness used by $\mathcal{C}), \mathcal{D}$ 's response computation as $b \leftarrow \operatorname{OPRF} \cdot \mathcal{D}(K, a)$, and $\mathcal{C}$ 's local computation of the final output as OPRF. $\mathcal{C}_{2}(b, r)$. We use the corresponding notation in the case of S-OPRF, except that S-OPRF.D takes as an input a triple $(K, i, a)$ where $i$ is an attribute and outputs a pair $(b, \rho)$. See below for a simple implementation of these procedures for the case of the Hashed Diffie-Hellman OPRF.

OSPIR-OXT Specification. With the above ingredients and notation we specify OSPIR-OXT on the basis of MC-OXT via the following changes.

Keys. Select key $K_{S}$ for $F_{G} ; K_{T}$ and $K_{X}$ for $F_{G}^{m} ; K_{I}$ for $F_{p}$; and $K_{M}$ for the authenticated encryption scheme.

EDBSetup. Follow the EDBSetup procedure of MC-OXT except for computing strap $\leftarrow$ $F_{G}\left(K_{S}, w\right)$ and $x \operatorname{tag} \leftarrow\left(F_{G}^{m}\left(K_{X}, w\right)\right)^{\text {xind }}$, and for implementing the TSetGetTag procedure as TSetGetTag $\left(K_{T}, w\right)=F_{G}^{m}\left(K_{T}, w\right)$, which means that we compute $\operatorname{stag}(w) \leftarrow F_{G}^{m}\left(K_{T}, w\right)$; index $\mathbf{t}(w)$ in $\mathbf{T}$ by $\operatorname{stag}(w)$ instead of by $w$ itself; and generate TSet using the modified API procedure TSetSetup' $(\mathbf{T})$.

GenToken protocol. This is the main change with respect to OSPIR-OXT; it follows the above mechanisms and is presented in Figure 2.

Search protocol. Same as MC-OXT except that stag is not included under env but rather it is provided to $\mathcal{E}$ by $\mathcal{C}$ as bstag from which $\mathcal{E}$ computes stag $\leftarrow$ (bstag $)^{1 / \rho_{1}}$. 
Instantiation via Hashed Diffie-Hellman OPRF. Our implementation and analysis of OSPIR-OXT assumes the use of Hashed DH OPRF mentioned above, namely, $F_{G}(K, x)=$ $(H(x))^{K}$. The instantiations of OPRF and S-OPRF protocols in this case are as follows. OPRF. $\mathcal{C}_{1}(x)$ and S-OPRF. $\mathcal{C}_{1}(x)$ both pick random $r$ in $Z_{p}^{*}$, set $a \leftarrow(H(x))^{r}$, and output $(a, r)$. Procedure OPRF.D $(K, a)$, where $K \in Z_{p}^{*}$ is a key for PRF $F_{G}$, outputs $b \leftarrow a^{K}$. Procedure S-OPRF.D $(K, i, a)$, where $i \in\{1, \ldots, m\}$ and $K=(K[1], \ldots, K[m]) \in\left(Z_{p}^{*}\right)^{m}$ is a key for PRF $F_{G}^{m}$, picks random $\rho$ in $Z_{p}^{*}$, computes $b \leftarrow a^{K[i] \cdot \rho}$, and outputs $(b, \rho)$. Procedures OPRF. $\mathcal{C}_{1}(b, r)$ and S-OPRF. $\mathcal{C}_{1}(b, r)$ both output $b^{1 / r}$. Note that if parties follow the protocol, $\mathcal{C}$ 's final output is equal to $(H(x))^{K}=F_{G}(K, x)$ in the OPRF protocol, while in the S-OPRF protocol it is equal to $(H(x))^{K[i] \cdot \rho}$, which is equal to $\left(F_{G}^{m}(K, x)\right)^{\rho}$ if $i=I(x)$. These OPRF and S-OPRF protocols emulate their corresponding ideal functionalities in ROM under so-called One-More Gap Diffie-Hellman assumption [21], see Section 5.

Figure 4 in Appendix B shows the OSPIR-OXT scheme instantiated with the above Hashed DH OPRF. It helps visualize the entire protocol and it reflects our actual implementation. In it we denote keys $K_{T}$ and $K_{X}$ of PRF $F_{G}^{m}$ by vectors of exponents in $Z_{p}^{*}$, respectively $\left(k_{1}, \ldots, k_{m}\right)$ and $\left(e_{1}, \ldots, e_{m}\right)$, where $m$ is the number of attributes. Also, because of the specific OPRF instantiation we equate $a_{s}$ to $a_{1}$ in $\mathcal{C}$ 's message of the GenToken protocol, instead of computing these two blinded versions of keyword $w_{1}$ separately, as in Figure 2.

\subsection{Supporting Boolean Queries}

For simplicity we presented our protocols for the case of conjunctions. The protocols can be readily adapted to search boolean queries in "searchable normal form (SNF)", i.e., of the form " $w_{1} \wedge \phi\left(w_{2}, \ldots, w_{m}\right)$ " (intended to return any document that matches keyword $w_{1}$ and in addition satisfies the formula $\phi$ on the remaining keywords). In this case, OXT and its derivatives change only in the way $\mathcal{E}$ determines which tuples match a query (i.e., which values e it sends back to $\mathcal{C}$ ). Specifically, in OXT the $c$-th tuple matches if and only if $x$ token $[c, i]^{y / \rho_{i}} \in \mathrm{XSet}$ for all $2 \leq i \leq n$. Instead, for boolean queries as above, $\mathcal{E}$ will have a set of boolean variables $v_{2}, \ldots, v_{n}$ and will set $v_{i}$ to the truth value of the predicate xtoken $[c, i]^{y / \rho_{i}} \in \mathrm{XSet}$. A tuple is matching if and only if the result of evaluating $\phi$ on these values returns true. The complexity of boolean search is same as for conjunctions, i.e., proportional to $\left|\mathrm{DB}\left(w_{1}\right)\right|$, and leakage to $\mathcal{E}$ is the same as for a conjunctive query on the same set of keywords except that $\mathcal{E}$ also learns the expression $\phi$ being evaluated. See [9] for details and support of other forms of boolean queries.

In the OSPIR-SSE setting, supporting boolean queries requires policies that are defined in terms of such queries. Specifically, a policy will determine a set of allowed pairs $(\psi, I)$ where $\psi$ is a symbolic boolean expression and $I$ a sequence of attributes, one per each variable in $\psi$. Thus, leakage to $\mathcal{D}$ will include $I$ (as in the case of conjunctions) plus the symbolic expression being evaluated. 


\subsection{Computational Cost}

Here we provide an operations count for OSPIR-OXT when instantiated with the DH-based OPRF noting its (mild) overhead over the original OXT protocol from [9]. The computational cost of OSPIR-OXT is easy to quantify by inspecting Figure 4 in Appendix B.

The cost of pre-processing (EDBSetup) is dominated by operations related to the group $G$, mainly exponentiations: For every $w \in \mathrm{W}$ :

1. One hashing operation, $H(w)$, of keyword $w$ into an element of the group $G$.

2. Two exponentiations: $\operatorname{strap}(w)=(H(w))^{K_{S}}$ and $\operatorname{stag}(w)=(H(w))^{k_{i}}$.

3. For every ind in $\mathrm{DB}(w)$ : One exponentiation $H(w)^{e_{i} \cdot \text { xind }}$ for computing an XSet element.

The first two items are specific to OSPIR-OXT while the third is from the original OXT protocol, except that here the base for exponentiation is changed from the generator $g$ in OXT to the value $H(w)$. Hence the overhead introduced by OSPIR-OXT is given by the first two items and the variable base. Importantly, the overhead for the first two items is only linear in $|\mathrm{W}|$, typically much smaller than the number of exponentiations in OXT (item 3), namely one per pair ( $w$, ind) for ind $\in \operatorname{DB}(w)$. As for the latter exponentiations, while the bases are variable, each $H(w)$ is typically used with a very large number of exponentiations (as the number of documents containing $w$ ) hence allowing for significant same-base optimizations. The hashing of $w$ into the group $G$ (item 1 ) is modeled as a random oracle, hence it rules out algebraic implementations such as $g^{h(w)}$. For the elliptic curves groups we use, $H$ is realized by sampling a field element $e$ and a bit $b$ from a PRNG seeded with $w$ until $(e, b)$ is the compressed representation of a valid group element. Depending on the particular nature of a curve, the generic square root algorithms required in solving the Weierstrass equation, though, can be extremely inefficient: Our original implementation for the chosen NIST 224p curve using OpenSSL's standard algorithms was more than an order of magnitude slower than a normal exponentiation and considerably more when common-base optimization is used. By implementing our own algorithm inspired by [4] and optimizing it for this particular field, we could reduce the cost down to the order of an exponentiation. Once all these optimizations are in place, the performance of OSPIR-OXT is remarkable as shown in Section 4.4 and, in much more details, in a companion paper [8].

The dominating cost of GenToken is just $2 n+1$ exponentiations for the client and $n+1$ for $\mathcal{D}$.

Finally, the cost of query processing between $\mathcal{C}$ and $\mathcal{E}$ (Search) on a $n$-term SNF expression is as follows:

1. $\mathcal{C}$ computes $n-1$ exponentiations for each tuple in $\operatorname{TSet}\left(w_{1}\right)$

2. $\mathcal{E}$ performs up to $n-1$ exponentiations for each element in $\operatorname{TSet}\left(w_{1}\right)$. 
Note that $\mathcal{C}$ can apply same-base optimization to the exponentiations since each term in the SNF expression has its own fixed base. On the other hand, $\mathcal{E}$ cannot use same-base optimizations. However, note that as soon as one of the values xtoken $[c, i]^{y / \rho_{i}}$ for a conjunction is found not to be in XSet, the other terms for this conjunction do not have to be evaluated (hence avoiding the need for these exponentiations). Similarly, for general Boolean expressions, early termination can be exploited to reduce costly computation. This highlights an important optimization for query processing (especially for queries with large TSet $\left(w_{1}\right)$ sets): Besides choosing the s-term as a term with high-entropy to keep TSet $\left(w_{1}\right)$ small, also choose the evaluation order of x-terms of an SNF expression such that it maximizes the probability of early termination and, hence, reduces the number of (expected) exponentiations executed by $\mathcal{E}$, e.g., for conjunctions order the x-terms in descending order of entropy (equivalently, ascending order of frequency).

Note (frequency ordering). In the OSPIR setting the client may not know the frequency of terms in the database and $\mathcal{D}$ will not know the query values to choose such terms optimally. Thus, the exact mechanism for determining the above ordering will depend on the specific setting. In our implementation we decide on ordering based on typical entropy of attributes; e.g., assuming last names have more entropy than names, and names more entropy than addresses, etc. (note that an attribute-based ordering is more privacy-preserving for the client than a value-based one).

\subsection{Implementation and performance}

The practicality of the proposed schemes was validated by experiments on DBs which included e.g. English-language Wikipedia (13,284,801 records / 2,732,311,945 indexed tuples), and a synthetic US census database (100 million records / 22,525,274,592 index tuples, resulting in EDB with 1.7 TB TSet and 0.4 TB XSet). To illustrate search efficiency, in the census DB case we executed complex queries like

$$
\begin{aligned}
& \text { SELECT ID WHERE FNAME='CHARLIE' AND SEX='FEMALE' AND NOT } \\
& (\text { STATE='NY' OR STATE='MA' OR STATE='PA' OR STATE='NJ) }
\end{aligned}
$$

in about 4 seconds on an IBM Blade dual Intel 4-core Xeon processor and storage provided by a (low-end) 6.2TB RAID-5 storage system. Preprocessing of such large DBs (TSet and XSet creation) has been feasible by, among other things, optimization of common-base exponentiations, achieving aggregated (parallel) rate of about 500,000 exp.'s/sec. for the NIST 224p elliptic curve.

See [8] for details on implementation and performance as well as for the extension of OXT and OSPIR-OXT to support dynamic databases (where documents can be added, deleted and modified). 


\section{Security}

We analyze security of the OSPIR-SSE scheme. We focus on the OSPIR case as it is the more comprehensive setting and it contains MC-SSE as a special case. The SSE-OXT protocol is analyzed in the SSE setting in [9].

\subsection{Extended Notion of TSet Correctness.}

As we mentioned in Section 2, in the context of MC-SSE and OSPIR-SSE setting the correctness property of the TSet datastructure must be extended to include the (lack of) false positives in addition to the false negatives. Intuitively, whereas in all SSE settings we must assure that the metadata $\mathbf{T}[w]$ associated with keyword $w$ can be recovered in its entirety given the $\operatorname{stag}(w)$ search handle, in the MC-SSE and OSPIR-SSE settings we must in addition assure that no data is returned without an explicitely provided search handle. To simplify stating and using the latter property we restrict it to the case where TSetGetTag is a deterministic function, i.e. where for each $K_{T}$, w there exists a unique stag $=\operatorname{TSetGetTag}\left(K_{T}, w\right)$.

Formally, we call a T-set scheme (computationally) correct if for every $\mathbf{T}$ and every efficient algorithm $A$, the following two facts hold, reflecting negligible probability of false negatives and false positives, respectively. First, there must be at most negligible probability that $\mathbf{t} \neq \mathbf{T}[w]$ in the experiment where (TSet, $\left.K_{T}\right) \leftarrow$ TSetSetup $(\mathbf{T}), w \leftarrow A\left(\mathbf{T}\right.$, TSet, $\left.K_{T}\right)$, stag $\leftarrow$ TSetGetTag $\left(K_{T}, w\right)$, and $\mathbf{t} \leftarrow$ TSetRetrieve(TSet, stag). Secondly, there must be at most negligible probability that $b=1$ in an experiment where (TSet, $\left.K_{T}\right) \leftarrow$ TSetSetup $(\mathbf{T})$, stag $^{*} \leftarrow \operatorname{Adv}\left(\mathbf{T}\right.$, TSet, $\left.K_{T}\right), \mathbf{t} \leftarrow$ TSetRetrieve(TSet, stag*), and we assign $b \leftarrow 1$ if it holds that $\mathbf{t}$ is non-empty and $\operatorname{stag}^{*} \neq \operatorname{TSetGetTag}\left(K_{T}, w\right)$ for all $w \in \mathbf{W}$; otherwise $b \leftarrow 0$.

It is easy to see that this extended correctness notion is satisfied by TSet implementations of both [9] and [8].

\subsection{OSPIR-SSE Security and Correctness Definitions}

SSE security definitions where the only adversarial entity is server $\mathcal{E}$ are provided in prior work. Here we follow the definitions from [9] - which in turn follow [11, 13] - and extend them to the MC setting by considering multiple malicious clients and to the OSPIR setting by adding also the data owner $\mathcal{D}$ as an adversarial entity. All security definitions follow the ideal/real model framework of secure computation and are parametrized by a leakage function $\mathcal{L}$ bounding the information leaked to an adversarial party in addition to the intended output for that party. Specifically, we ask that whatever an adversary can do by running the real protocol on data and queries chosen by the adversary, a simulator can do solely on the basis of the leakage function. 
Correctness. We say that an OSPIR-SSE scheme $\Sigma=$ (EDBSetup, GenToken, Search) is computationally correct if for every efficient algorithm $A$, there is a negligible probability that the following experiment outputs 0 . On inputs (DB, RDK) and $\bar{w}^{(1)}, \ldots, \bar{w}^{(m)}$ provided by $A$, execute $(K, \mathrm{EDB}) \leftarrow \operatorname{EDBSetup}(\mathrm{DB}, \mathrm{RDK})$; and for $i=1,2, \ldots$, execute protocol GenToken on $\mathcal{C}$ 's input $\bar{w}^{(i)}$ and $\mathcal{D}$ 's inputs $(\mathrm{P}, K)$, denote $\mathcal{C}$ 's output as token ${ }^{(i)}$, execute protocol Search between $\mathcal{C}$ on input token ${ }^{(i)}$ and $\mathcal{E}$ on input EDB and denote $\mathcal{C}$ 's outputs as a pair $\left(\right.$ indSet $\left.^{(\mathrm{i})}, \operatorname{rdkSet}^{(\mathrm{i})}\right)$. Output 1 if for each $i$ we have that indSet ${ }^{(\mathrm{i})}=\mathrm{DB}\left(\overline{\mathrm{w}}^{(\mathrm{i})}\right)$ and $\operatorname{rdkSet}^{(i)}=\operatorname{RDK}\left[\operatorname{DB}\left(\overline{\mathrm{w}}^{(\mathrm{i})}\right)\right]$. Otherwise output 0 .

Security against adversarial server $\mathcal{E}$. Security against adversarial (honest-but-curious) $\mathcal{E}$ has been the focus of prior SSE work. Adapting the definition of $\mathcal{L}$-semantic security against adaptive attacks (by the server $\mathcal{E}$ ) from [9] to our setting is straightforward and is omitted here.

Security against adversarial clients. The definition captures the information leaked to a malicious client in addition to the intended output $\mathrm{DB}(\bar{w})$ and the corresponding recorddecrypting keys $\operatorname{RDK}[\mathrm{DB}(\bar{w})]$. The definition compares the real execution to an emulation of an interaction with algorithm I-SSE $E_{\mathcal{L}}$, which models an ideal functionality of the OSPIRSSE scheme instantiated with the leakage function $\mathcal{L}$. The interactive algorithm I-SSE ${ }_{\mathcal{L}}$, running on local input (DB, RDK, $\mathrm{P}$ ), answers queries $\bar{w} \in \mathrm{W}^{*}$ by checking if av $(\bar{w}) \in \mathrm{P}$. If the check verifies, then it replies to this $\bar{w}$ with a triple $(\operatorname{DB}(\bar{w}), \operatorname{RDK}[\operatorname{DB}(\bar{w})], \mathcal{L}(\mathrm{DB}, \bar{w}))$, and if $\operatorname{av}(\bar{w}) \notin \mathbf{P}$ then it sends back a rejection symbol $\perp$.

Definition 1 Let $\Pi=$ (EDBSetup, GenToken,Search) be an OSPIR-SSE scheme. Given algorithms $\mathcal{L}, A$, and $S=\left(S_{0}, S_{1}, S_{2}\right)$ we define experiments (algorithms) $\operatorname{Real}_{A}^{\Pi}(\lambda)$ and Ideal $_{A, S}^{\Pi}(\lambda)$ as follows:

$\operatorname{Real}_{A}^{\Pi}(\lambda): A\left(1^{\lambda}\right)$ chooses (DB, RDK, P), and the experiment runs $(K, \mathrm{EDB}) \leftarrow$ EDBSetup (DB, RDK). Adversary $A$ can then adaptively invoke instances of the protocol GenToken and Search, interacting with party $\mathcal{D}$ running on input $K$ and $\mathrm{P}$ in the first case and with party $\mathcal{E}$ running on input EDB in the second case. Note that $A$ can behave arbitrarily in all these protocol instances. Let $q$ be the number of GenToken instances and let $\mathrm{av}_{i}$ be $\mathcal{D}$ 's local output in the $i$-th instance. If at any point $A$ halts and outputs a bit $b$, the game outputs $\left(b, \mathrm{av}_{1}, \ldots, \mathrm{av}_{q}\right)$.

Ideal $_{A, S}^{\Pi}(\lambda)$ : $A\left(1^{\lambda}\right)$ chooses (DB, RDK, P) as above, while the experiment initializes $S=$ $\left(S_{0}, S_{1}, S_{2}\right)$ by running st $\leftarrow S_{0}\left(1^{\lambda}\right)$. Subsequently, each time $A$ invokes an instance of protocol GenToken, it interacts with the experiment running $S_{1}(\mathrm{st}, \mathrm{P})$, whereas if $A$ invokes an instance of protocol Search, it interacts with the experiment running $S_{2}(\mathrm{st})$. Both $S_{1}$ and $S_{2}$ algorithms are allowed to update the global simulator's state st while interacting with $A$. Both can issue queries $\bar{w}$ to $\mathrm{I}-\mathrm{SSE}_{\mathcal{L}}(\mathrm{DB}, \mathrm{RDK}, \mathrm{P})$. Let $q$ be the number of these queries and let $\mathrm{av}_{i}=I\left(\bar{w}_{i}\right)$, where $\bar{w}_{i}$ is the $i$-th query. As above, if at any point $A$ halts and output a bit $b$, the game outputs $\left(b, \mathrm{av}_{1}, \ldots, \mathrm{av}_{q}\right)$. 
We call $\Pi \mathcal{L}$-semantically-secure against malicious clients if for any efficient algorithm $A$ there is an efficient algorithm $S$ s.t. the statistical difference between tuples $\left(b, \mathrm{av}_{1}, \ldots, \mathrm{av}_{q}\right)$ output by experiments $\mathbf{R e a l}_{A}^{\Pi}$ and $\mathbf{I d e a l}{ }_{A, S}^{\Pi}$ is a negligible function of the security parameter $\lambda$.

Security against adversarial data owner. Security against a data-owner $\mathcal{D}$ models privacy of the client's queries $\bar{w}$ against malicious $\mathcal{D}$, given an adaptive choice of the client's queries. Similarly to the case of security against either the client $\mathcal{C}$ or the EDB-storing server $\mathcal{E}$, this security definition also allows for leakage of some information $\mathcal{L}(\bar{w})$ regarding the query $\bar{w}$ to $\mathcal{D}$.

Definition 2 Let $\Pi=$ (EDBSetup, GenToken, Search) be an OSPIR-SSE scheme. Given algorithms $\mathcal{L}, A$, and $S$ we define experiments (algorithms) $\operatorname{Real}_{A}^{\Pi}(\lambda)$ and $\operatorname{Ideal}_{A, S}^{\Pi}(\lambda)$ as follows:

$\operatorname{Real}_{A}^{\Pi}(\lambda)$ : Adversary $A\left(1^{\lambda}\right)$ can adaptively invoke any number of GenToken instances by specifying a query $\bar{w}$ and interacting with party $\mathcal{C}$ running the GenToken protocol on input $\bar{w}$. At any point $A$ can halt and output a bit, which the game uses as its own output.

Ideal $_{A, S}^{\Pi}(\lambda)$ : Adversary $A\left(1^{\lambda}\right)$ can adaptively invoke any number of GenToken instances as above, but for any query $\bar{w}$ which $A$ specifies, it interacts with $S$ running on input $\mathcal{L}(\bar{w})$. As above if $A$ halts and output a bit, the game uses this bit as its own output.

$W e$ call $\Pi \mathcal{L}$-semantically-secure against malicious data owner if for any efficient alg. $A$ there is an efficient alg. $S$ s.t. $\operatorname{Pr}\left[\operatorname{Real}_{A}^{\Pi}(\lambda)=1\right]-\operatorname{Pr}\left[\operatorname{Ideal}_{A, S}^{\Pi}(\lambda)=1\right] \leq \operatorname{neg}(\lambda)$.

Note (non-collusion between $\mathcal{D}$ and $\mathcal{E}$ ). We stress that even though the data owner $\mathcal{D}$ can be arbitrarily malicious, we assume that $\mathcal{D}$ and $\mathcal{E}$ do not collude. Indeed, client's security in the OSPIR-OXT scheme we propose is not maintained against such collusion. Moreover, providing query-privacy from $\mathcal{D}$ under collusion with $\mathcal{E}$ would have the (impractical) cost of a single-server symmetric PIR protocol. The hospital example mentioned in the introduction, is a case where such non-collusion requirement makes sense. Indeed, it is the hospital interest not to learn the queries: It helps avoiding liability and complying with regulations as well as withstanding potential insider attacks. Similarly, for a service providing private access to a database (e.g. to a patent repository) preserving client privacy is part of its very business model. See also [18].

\subsection{Security of OSPIR-OXT}

Correctness of OSPIR-OXT. We first argue that protocol OSPIR-OXT is correct. Assuming a computationally correct TSet implementation (see Section 2.1), any correctness 
errors can only come from collisions in PRF functions, including function $F_{K_{X}, K_{I}}$ effectively used in computing xtag values, defined as $F_{K_{X}, K_{I}}(w$, ind $)=\left(F_{G}^{m}\left(K_{X}, w\right)\right)^{F_{p}\left(K_{I} \text {,ind }\right)}$. But assuming the PRF property of $F_{p}$, and the PRF property of $F_{G}^{m}$, which holds under DDH in ROM, function $F_{K_{X}, K_{I}}$ is a PRF too, and so collision probability is negligible, resulting in negligible error probability over the execution of the OSPIR-OXT correctness experiment. Thus, we have:

Theorem 3 The OSPIR-SSE scheme OSPIR-OXT instantiated with the Hashed DiffieHellman OPRF is computationally correct assuming that the DDH assumption holds, that the T-set implementation is computationally correct, that $F_{p}$ is a secure PRF, and assuming the Random Oracle Model for hash function $H$.

Security of OSPIR-OXT. Using the security notions explained above we describe the security properties of the OSPIR-SSE scheme OSPIR-OXT instantiated with the Hashed Diffie-Hellman OPRF, as shown in Figure 4 in Appendix B. We first state the OM-GDH security assumption required for the security of the OPRF and S-OPRF sub-protocols of this OSPIR-OXT instantiation.

One-More Gap Diffie-Hellman (OM-GDH). Let $G=G_{\lambda}$ be a prime order cyclic group of order $p=p(\lambda)$ generated by $g$. We say that the One-More Gap Diffie-Hellman $(O M-G D H)$ assumption holds in $G$ if $\mathbf{A d v}_{G, A}^{\mathrm{ddh}}(\lambda)$ is negligible for all efficient adversaries $A$, where $\operatorname{Adv}_{G, A}^{\mathrm{ddh}}(\lambda)$ is defined as the probability that $A$ wins the following game: (1) The game chooses random $t$ in $Z_{p}^{*}$ and two random elements $h_{1}, h_{2}$ in $G ;(2) A$, on input $h_{1}, h_{2}$, specifies a single query $a$ to the Diffie-Hellman oracle, which on input $a$ returns $b \leftarrow a^{t}$; (3) $A$ can make any number of queries to a Decisional Diffie-Hellman oracle $\mathrm{DDH}_{\mathrm{t}}(\cdot, \cdot)$, which on input $(h, v)$ returns 1 if $v=h^{t}$ and 0 otherwise; (4) Finally $A$ outputs two values $v_{1}, v_{2}$, and we say that $A$ wins the game if $v_{1}=\left(h_{1}\right)^{t}$ and $v_{2}=\left(h_{2}\right)^{t}$.

Security against adversarial server $\mathcal{E}$. The OSPIR-SSE scheme OSPIR-OXT is $\mathcal{L}_{\text {oxt }}{ }^{-}$ semantically-secure against adaptive server $\mathcal{E}$ under the same assumptions and for the same leakage function $\mathcal{L}_{\text {oxt }}$ as the underlying SSE scheme OXT of [9]. This is because the specific PRF's used by OSPIR-OXT in EDB construction are instantiations of general PRF's considered in OXT, and because $\mathcal{E}$ 's view of the Search protocol in the OSPIR-OXT scheme can be generated from $\mathcal{E}$ 's view of Search in the OXT scheme. Specifically, each $\rho_{i}$ in env is random in $Z_{p}^{*}$, and bstag and each bxtoken $[c, i]$ value in Figure 4 (Appendix B) can be computed by exponentiating values stag and xtoken $[c, i]$ in Figure 1 to, respectively, $\rho_{1}$ and $\rho_{i}$.

Security against adversarial client $\mathcal{C}$. Let Mask $\left(\left|\mathrm{DB}\left(w_{1}\right)\right|\right)$ denote an upper bound on $\left|\mathrm{DB}\left(w_{1}\right)\right|$ used by $\mathcal{E}$ to mask the size of $\operatorname{TSet}\left(w_{1}\right)$ when responding to $\mathcal{C}$ 's queries as described at the end of Section 3. 
Theorem 4 Let $\mathcal{L}$ be a defined as $\mathcal{L}(\mathrm{DB}, \bar{w})=\operatorname{Mask}\left(\left|\mathrm{DB}\left(w_{1}\right)\right|\right)$ for $\bar{w}=\left(w_{1}, \ldots, w_{n}\right)$. OSPIR-SSE scheme OSPIR-OXT instantiated with the Hashed Diffie-Hellman OPRF is $\mathcal{L}$-semantically-secure against malicious clients assuming that the One-More Gap DiffieHellman assumption holds in $G$, that $F_{p}$ is a secure PRF, that the T-set implementation is (computationally) correct, that (AuthEnc, AuthDec) is an IND-CPA and Strongly-UF-CMA authenticated encryption scheme, and assuming the Random Oracle Model for hash function $H$.

Proof: The proof is lengthy and is presented in Appendix A.

Security against adversarial data owner $\mathcal{D}$. In our OSPIR-SSE scheme a malicious $\mathcal{D}$ learns nothing about clients' query $\bar{w}=\left(w_{1}, \ldots, w_{n}\right)$ except for the vector of attributes $\operatorname{av}(\bar{w})=\left(I\left(w_{1}\right), \ldots, I\left(w_{n}\right)\right)$.

Theorem 5 Let $\mathcal{L}$ be a leakage function defined as $\mathcal{L}(\bar{w})=\operatorname{av}(\bar{w})$. OSPIR-SSE scheme OSPIR-OXT instantiated with the Hashed Diffie-Hellman OPRF is $\mathcal{L}$-semantically-secure against malicious data owner.

Proof: The view of $\mathcal{D}$ in the GenToken protocol of OSPIR-OXT (Figure 2) consists of the attribute vector $\operatorname{av}(\bar{w})=\left(I_{1}, \ldots, I_{n}\right)$ corresponding to the query $\bar{w}$ and the values $a_{s}, a_{1}$, $\ldots, a_{n}$ where $a_{s}$ is output by OPRF. $\mathcal{C}_{1}\left(w_{1}\right)$ and each $a_{i}$ is output by S-OPRF.Charlie $1\left(w_{i}\right)$. In the Hashed DH OPRF instantiation of this scheme in Figure 4 (Appendix B), these values are formed as $a_{j} \leftarrow H\left(w_{j}\right)^{r_{j}}$ for random $r_{j}$ 's in $Z_{p}^{*}$ (additionally, $a_{s}$ is set to $a_{1}$ ). Since $G$ is of prime order, every element in $G \backslash\{1\}$ is a generator, and thus each $a_{j}$ is uniform in $G$. Thus, it is straightforward to simulate $\mathcal{D}$ 's view of the GenToken protocol from $\mathcal{L}(\bar{w})=\operatorname{av}(\bar{w})$.

\subsection{Extensions: Reducing Leakage to $\mathcal{D}$}

In the full version we show how to adapt OSPIR-OXT to a setting where a third party, called a policy manager, authorizes queries while $\mathcal{D}$ can enforce them without learning the policy, the boolean expression or the queried attributes; only the number of such attributes is learned by $\mathcal{D}$. This setting is precisely what is needed to implement searches authorized by a warrant while keeping the searched information hidden from all parties except the authorized searcher.

In addition, OSPIR-OXT can be extended (even without introducing a policy manager) so that the leakage about queried attributes to $\mathcal{D}$ is further limited to the minimum needed to make policy decisions (e.g., $\mathcal{D}$ may not need to know the exact attributes in a query but only the attribute classes they belong to). 


\section{Acknowledgment}

Supported by the Intelligence Advanced Research Projects Activity (IARPA) via Department of Interior National Business Center (DoI / NBC) contract number D11PC20201. The U.S. Government is authorized to reproduce and distribute reprints for Governmental purposes notwithstanding any copyright annotation thereon. Disclaimer: The views and conclusions contained herein are those of the authors and should not be interpreted as necessarily representing the official policies or endorsements, either expressed or implied, of IARPA, DoI/NBC, or the U.S. Government.

\section{References}

[1] M. Abdalla, M. Bellare, D. Catalano, E. Kiltz, T. Kohno, T. Lange, J. Malone-Lee, G. Neven, P. Paillier, and H. Shi. Searchable encryption revisited: Consistency properties, relation to anonymous IBE, and extensions. In V. Shoup, editor, CRYPTO 2005, volume 3621 of $L N C S$, pages 205-222. Springer, Aug. 2005.

[2] L. Ballard, S. Kamara, and F. Monrose. Achieving efficient conjunctive keyword searches over encrypted data. In S. Qing, W. Mao, J. López, and G. Wang, editors, ICICS 05, volume 3783 of LNCS, pages 414-426. Springer, Dec. 2005.

[3] M. Bellare, A. Boldyreva, and A. O'Neill. Deterministic and efficiently searchable encryption. In A. Menezes, editor, CRYPTO 2007, volume 4622 of LNCS, pages 535552. Springer, Aug. 2007.

[4] D. J. Bernstein. Faster square roots in annoying finite fields. http://cr.yp.to/ papers/sqroot.pdf, 2001.

[5] D. Boneh, G. Di Crescenzo, R. Ostrovsky, and G. Persiano. Public key encryption with keyword search. In C. Cachin and J. Camenisch, editors, EUROCRYPT 2004, volume 3027 of LNCS, pages 506-522. Springer, May 2004.

[6] D. Boneh and B. Waters. Conjunctive, subset, and range queries on encrypted data. In S. P. Vadhan, editor, TCC 2007, volume 4392 of LNCS, pages 535-554. Springer, Feb. 2007.

[7] J. W. Byun, D. H. Lee, and J. Lim. Efficient conjunctive keyword search on encrypted data storage system. In EuroPKI, pages 184-196, 2006.

[8] D. Cash, J. Jaeger, S. Jarecki, C. Jutla, H. Krawczyk, M.-C. Roşu, and M. Steiner. Dynamic Searchable Encryption in Very Large Databases: Data Structures and Implementation. 21st Annual Network and Distributed System Security Symposium, NDSS 2014, San Diego, 2014., 2014. 
[9] D. Cash, S. Jarecki, C. Jutla, H. Krawczyk, M. Rosu, and M. Steiner. Highly-scalable searchable symmetric encryption with support for boolean queries. Crypto'2013. Cryptology ePrint Archive, Report 2013/169, Mar. 2013. http://eprint.iacr.org/2013/ 169.

[10] Y.-C. Chang and M. Mitzenmacher. Privacy preserving keyword searches on remote encrypted data. In J. Ioannidis, A. Keromytis, and M. Yung, editors, ACNS 05, volume 3531 of $L N C S$, pages 442-455. Springer, June 2005.

[11] M. Chase and S. Kamara. Structured encryption and controlled disclosure. In ASIACRYPT 2010, LNCS, pages 577-594. Springer, Dec. 2010.

[12] E. D. Cristofaro, Y. Lu, and G. Tsudik. Efficient techniques for privacy-preserving sharing of sensitive information. In J. M. McCune, B. Balacheff, A. Perrig, A.-R. Sadeghi, A. Sasse, and Y. Beres, editors, TRUST, volume 6740 of Lecture Notes in Computer Science, pages 239-253. Springer, 2011.

[13] R. Curtmola, J. A. Garay, S. Kamara, and R. Ostrovsky. Searchable symmetric encryption: improved definitions and efficient constructions. In A. Juels, R. N. Wright, and S. Vimercati, editors, ACM CCS 06, pages 79-88. ACM Press, Oct. / Nov. 2006.

[14] M. J. Freedman, Y. Ishai, B. Pinkas, and O. Reingold. Keyword search and oblivious pseudorandom functions. In J. Kilian, editor, TCC 2005, volume 3378 of LNCS, pages 303-324. Springer, Feb. 2005.

[15] E.-J. Goh. Secure indexes. Cryptology ePrint Archive, Report 2003/216, 2003. http: //eprint.iacr.org/.

[16] P. Golle, J. Staddon, and B. R. Waters. Secure conjunctive keyword search over encrypted data. In M. Jakobsson, M. Yung, and J. Zhou, editors, ACNS 04, volume 3089 of $L N C S$, pages 31-45. Springer, June 2004.

[17] Y. Huang and I. Goldberg. Outsourced private information retrieval with pricing and access control. Technical Report 2013-11, Centre for Applied Cryptographic Research (CACR), University of Waterloo, Feb. 2013.

[18] IARPA. Security and Privacy Assurance Research (SPAR) Program - BAA, 2011. http://www.iarpa.gov/solicitations_spar.html/.

[19] M. Islam, M. Kuzu, and M. Kantarcioglu. Access pattern disclosure on searchable encryption: Ramification, attack and mitigation. In Proceedings of the Symposium on Network and Distributed Systems Security (NDSS 2012), San Diego, CA, Feb. 2012. Internet Society.

[20] S. Jarecki and X. Liu. Efficient oblivious pseudorandom function with applications to adaptive OT and secure computation of set intersection. In O. Reingold, editor, TCC 2009, volume 5444 of $L N C S$, pages 577-594. Springer, Mar. 2009. 
[21] S. Jarecki and X. Liu. Fast secure computation of set intersection. In SCN 10, LNCS, pages 418-435. Springer, 2010.

[22] S. Kamara and K. Lauter. Cryptographic cloud storage. In Financial Cryptography Workshops, pages 136-149, 2010.

[23] S. Kamara, C. Papamanthou, and T. Roeder. Dynamic searchable symmetric encryption. In Proc. of CCS'2012, 2012.

[24] K. Kurosawa and Y. Ohtaki. UC-secure searchable symmetric encryption. In Financial Cryptography, page 285, 2012.

[25] M. Naor and O. Reingold. Number-theoretic constructions of efficient pseudo-random functions. In 38th FOCS, pages 458-467. IEEE Computer Society Press, Oct. 1997.

[26] V. Pappas, B. Vo, F. Krell, S. G. Choi, V. Kolesnikov, A. Keromytis, and T. Malkin. Blind Seer: A Scalable Private DBMS. Manuscript, 2013.

[27] E. Shi, J. Bethencourt, H. T.-H. Chan, D. X. Song, and A. Perrig. Multi-dimensional range query over encrypted data. In 2007 IEEE Symposium on Security and Privacy, pages 350-364. IEEE Computer Society Press, May 2007.

[28] D. X. Song, D. Wagner, and A. Perrig. Practical techniques for searches on encrypted data. In 2000 IEEE Symposium on Security and Privacy, pages 44-55. IEEE Computer Society Press, May 2000.

[29] P. van Liesdonk, S. Sedhi, J. Doumen, P. H. Hartel, and W. Jonker. Computationally efficient searchable symmetric encryption. In Proc. Workshop on Secure Data Management (SDM), pages 87-100, 2010.

[30] B. R. Waters, D. Balfanz, G. Durfee, and D. K. Smetters. Building an encrypted and searchable audit log. In NDSS 2004. The Internet Society, Feb. 2004.

[31] WSJ. U.S. Terrorism Agency to Tap a Vast Database of Citizens. Wall Street Journal 12/13/12. http://alturl.com/ot72x.

\section{A Proof of Theorem 4: Security Against Malicious Clients}

Here we prove Theorem 4 formulated in Page 22.

The simulator algorithm $S=\left(S_{0}, S_{1}, S_{2}\right)$ for this security proof is shown in Figure 3. Let $\Pi$ denote the scheme OSPIR-OXT instantiated with the Hashed Diffie-Hellman PRF, let $A$ be an adversary's algorithm and let $G_{0}$ denote the experiment $\mathbf{R e a l}_{A}^{\Pi_{1}}(\lambda)$, which models $A$ 's interaction with the real scheme OSPIR-OXT. We will denote by $G_{i}$ a sequence of modifications of $G_{0}$, s.t. the last game, $G_{12}$ is identical to the experiment $\operatorname{Ideal}_{A, S}^{\Pi}(\lambda)$. Let 
$\underline{S_{0}\left(1^{\tau}\right)}$

- Select key $K_{S}$ for $F_{G}, K_{X}$ and $K_{T}$ for $F_{G}^{m}, K_{P}$ for $F_{p}$, and $K_{M}$ for authenticated encryption.

- Initialize an empty table QList, which will be indexed by ciphertexts env, and a table TList, indexed by keywords $w$ in $\mathbf{W}$, which initially holds an empty set for each $w$.

- Set st $\leftarrow\left(K_{S}, K_{X}, K_{T}, K_{P}, K_{M}\right.$, QList, TList $)$.

$S_{1}($ st, P)

- On input $\left(a_{s}, a_{1}, \ldots, a_{n}\right),\left(I_{1}, \ldots, I_{n}\right)$ from $A$, abort if $\left(I_{1}, \ldots, I_{n}\right) \notin \mathrm{P}$.

- Pick $\rho_{i}^{\prime}, \rho_{i} \stackrel{\$}{\leftarrow} Z_{p}^{*}$ for each $i=1, \ldots, n$.

- Set $b_{s} \leftarrow\left(a_{s}\right)^{K_{S}}, b_{1} \leftarrow\left(a_{1}\right)^{K_{T}\left[I_{1}\right] \cdot \rho_{1}}$, and $b_{i} \leftarrow\left(a_{i}\right)^{K_{X}\left[I_{i}\right] \cdot \rho_{i}}$ for $i=2, \ldots, n$.

- Update QList in st by setting QList(env) $\leftarrow\left(I_{1}, \ldots, I_{n}, \rho_{1}, \ldots, \rho_{n}\right)$.

- Set env $\leftarrow \operatorname{AuthEnc}\left(K_{M},\left(\rho_{1}^{\prime}, \ldots, \rho_{n}^{\prime}\right)\right)$ and output (env, $\left.b_{s}, b_{1}, \ldots, b_{n}\right)$.

$S_{2}(\mathrm{st})$, on message (env, bstag, xtoken $[1]$, xtoken $\left.[2], \ldots\right)$ from $A$ :

- Retrieve $\left(I_{1}, \ldots, I_{n}, \rho_{1}, \ldots, \rho_{n}\right) \leftarrow$ QList(env). Abort if QList(env) $=\perp$.

- Set stag $\leftarrow(\text { bstag })^{1 / \rho_{1}}$. If there exists $w_{1} \in \mathrm{W}$ s.t. stag $=\left(H\left(w_{1}\right)\right)^{K_{T}\left[I_{1}\right]}$ and $I\left(w_{1}\right)=I_{1}$ then set strap $\leftarrow F_{G}\left(K_{S}, w_{1}\right)$ and $\left(K_{z}, K_{e}\right) \leftarrow\left(F_{\tau}(\operatorname{strap}, 1), F_{\tau}\left(\right.\right.$ strap, 2)). Abort if no such $w_{1}$ found.

- Set $c \leftarrow 0$ and found $\leftarrow F$, and perform the following loop while found $=F$.

- Set $c \leftarrow c+1$ and $z_{c} \leftarrow F_{p}\left(K_{z}, c\right)$. For xtoken $[c]=(x \operatorname{token}[c, 2], \ldots, x$ token $[c, n])$, if $\exists\left(w_{2}, \ldots, w_{n}\right) \in$ $\mathrm{W}^{n-1}$ s.t. xtoken $[c, i]=\left(H\left(w_{i}\right)\right)^{K_{X}\left[I_{i}\right] \cdot z_{c} \cdot \rho_{i}}$ and $I\left(w_{i}\right)=I_{i}$, for $i=2, \ldots, n$, then set found $\leftarrow \mathrm{T}$.

- Abort if found $=\mathrm{F}$ and xtoken $[c]$ is the last element in $A$ 's message.

- Send $\bar{w}=\left(w_{1}, w_{2}, \ldots, w_{n}\right)$ to $\operatorname{I-SSE}_{\mathcal{L}}(\mathrm{DB}, \mathrm{RDK}, \mathrm{P})$ where $w_{1}, w_{2}, \ldots, w_{n}$ are the keywords found above. Since $\operatorname{av}(\bar{w})=\left(I_{1}, \ldots, I_{n}\right)$ is guaranteed to be included in $\mathrm{P}, S_{2}$ receives back $(\mathrm{DB}(\bar{w}), \operatorname{RDK}(\mathrm{DB}(\bar{w})), \mathrm{TSetL})$.

- Set $S^{\prime} \leftarrow \emptyset$ and $D \leftarrow \operatorname{DB}(\bar{w})$. $\forall$ ind $\in D$ s.t. $(c$, ind, e $) \in \operatorname{TList}\left(w_{1}\right)$, add $c$ to $S^{\prime}$ and delete ind from $D$.

- Pick $S$ as random $|D|$-element subset in $\{1, \ldots, \mathrm{TSetL}\} \backslash S^{\prime}$, and while $S$ is non-empty do:

- Remove a random element $c$ from $S$ and a random element ind from $D$.

- Set rind $\leftarrow P_{\tau}\left(K_{P}\right.$, ind $), \mathrm{rdk} \leftarrow \mathrm{RDK}($ ind $), \mathrm{e} \leftarrow \operatorname{Enc}\left(K_{e},(\right.$ rind $\left.\mid \mathrm{rdk})\right)$.

- Update TList in st by adding (c, ind, e) to TList $\left(w_{1}\right)$.

- Starting from the last counter $c$ encountered above, perform the following loop while $c \leq$ TSetL:

- Set $z_{c} \leftarrow F_{p}\left(K_{z}, c\right)$. If xtoken $[c, i]=\left(H\left(w_{i}\right)\right)^{K_{X}\left[I_{i}\right] \cdot z_{c} \cdot \rho_{i}}$ for each $i=2, \ldots, n$, and if there exists $\left(c\right.$, ind, e) in TList $\left(w_{1}\right)$ s.t. ind $\in \mathrm{DB}(\bar{w})$, then send e to $A$, and set $c \leftarrow c+1$.

- Send stop to $A$ and halt.

Figure 3: $S=\left(S_{0}, S_{1}, S_{2}\right)$ : Simulators For the SECURIty Proof of the OSPIR-OXT Protocol 
$p_{i}$ be the probability that game $G_{i}$ outputs 1 . We argue that the difference between $p_{0}$ and $p_{12}$ is a negligible function of the security parameter $\lambda$.

Games $G_{1}$ and $G_{2}$. In $G_{1}$ we modify game $G_{0}$ by adding an abort if any authenticated ciphertext env accepted by the server in the Search protocol has not been generated and signed by the data owner in the GenToken procedure. $p_{1} \approx p_{0}$ by Strong-UF-CMA unforgeability of the authenticated encryption scheme. In game $G_{2}$ we add an abort if ever two GenToken instances generate the same env ciphertext. $p_{2} \approx p_{1}$ e.g. because $\rho_{i}$ 's are generated at random from $Z_{p}^{*}$, and collision in the ciphertext implies collision in the plaintext.

Game $G_{3}$. We add an abort if for any two keywords $w, w^{\prime} \in \mathrm{W}$ s.t. $w^{\prime} \neq w$ and $e i$ ther $F_{G}^{m}\left(K_{T}, w^{\prime}\right)=F_{G}^{m}\left(K_{T}, w\right)$ or $F_{G}^{m}\left(K_{X}, w^{\prime}\right)=F_{G}^{m}\left(K_{X}, w\right)$. Such collisions occur with negligible probability because $K_{G}^{m}$ is a PRF, and therefore $p_{3} \approx p_{2}$.

Game $G_{4}$. In the EDBSetup procedure we skip the step when TSet is created from the array $\mathbf{T}$, and we change the way the game responds in the Search protocol. As in $G_{0}$, the game computes stag $\leftarrow$ bstag $^{1 / \rho_{1}}$, but then it searches through the keyword space $\mathrm{W}$ for $w_{1}$ s.t. stag $=F_{G}^{m}\left(K_{T}, w_{1}\right)$. If such $w_{1}$ is found it assigns $\mathbf{t} \leftarrow \mathbf{T}\left[w_{1}\right]$, and otherwise it aborts. Since in game $G_{3}$ we have eliminated collisions in function $F_{G}^{m}$, if stag computed above is equal to $\operatorname{stag}\left(w_{1}\right)$ for some $w_{1} \in \mathrm{W}$ then by the no-false-negatives part of the TSet correctness property the $\mathbf{t}$ retrieved in game $G_{3}$ via TSetRetrieve(TSet, stag) is the same as $\mathbf{T}\left[w_{1}\right]$ retrieved in game $G_{4}$, except for a negligible probability of error. On the other hand, if $\operatorname{stag} \neq \operatorname{stag}(w)$ for all $w \in \mathbf{W}$, then by the no-false-positives part of the TSet correctness property the $\mathbf{t}$ retrieved in game $G_{3}$ is an empty list just like in game $G_{4}$, again except for a negligible error probability. It follows that $p_{4} \approx p_{3}$.

Game $G_{5}$. Instead of encrypting $\left(\rho_{1}, \rho_{2}, \ldots, \rho_{n}\right)$ in env, $G_{5}$ encrypts a string of independent random values $\left(\rho_{1}^{\prime}, \rho_{2}^{\prime}, \ldots, \rho_{n}^{\prime}\right)$. In addition, game $G_{5}$ keeps an array QList indexed by ciphertexts env, and when servicing GenToken requests $G_{5}$ stores in QList(env) the list of attribute indexes $\left(I_{1}, \ldots, I_{n}\right)$ and the true blinding values $\left(\rho_{1}, \ldots, \rho_{n}\right)$ used in the GenToken instance which generated ciphertext env. Therefore in the Search procedure, instead of decrypting $\rho_{i}$ 's from env, which now encrypts independent random values, $G_{5}$ retrieves these $\rho_{i}$ 's from QList(env). $p_{5} \approx p_{4}$ by IND-CCA of the authenticated encryption, and because already in game $G_{2}$ the only env's which are accepted in Search are uniquely identifying some GenToken instance.

Game $G_{6}$. We modify the test for identifying $w_{1}$ given bstag and $\rho_{1}$ recovered from QList(env), to $w_{1}$ s.t. (bstag) $)^{1 / \rho_{1}}=\left(H\left(w_{1}\right)\right)^{K_{T}\left[I_{1}\right]}$ and $I\left(w_{1}\right)=I_{1}$. In other words, compared to $G_{5}, G_{6}$ ignores $w_{1}$ 's s.t. stag $=(\mathrm{bstag})^{1 / \rho_{1}}=\left(H\left(w_{1}\right)\right)^{K_{T}\left[I\left(w_{1}\right)\right]}$ but $I\left(w_{1}\right) \neq I_{1}$. We argue that the probability of finding such $w_{1}$ is negligible even against all-powerful adversary. Denote the exponent $K_{T}\left[I_{1}\right] \cdot \rho_{1}$ used in computing $b_{1}$ from $a_{1}$ in $j$-th GenToken instance as $t_{j}$. Note that each $t_{j}$ is uniformly random in $Z_{p}^{*}$. Using this notation, the way game $G_{6}$ tests each keyword $w_{1}$ given bstag in the Search procedure can be rewritten as testing whether bstag $=\left(H\left(w_{1}\right)\right)^{\left(K_{T}\left[I\left(w_{1}\right)\right] / K_{T}\left[I_{1}\right]\right) \cdot t_{j}}$ where $t_{j}$ is used in GenToken instance that generated 
ciphertext env used in this Search instance. In particular, the discrete logarithm between $\left(H\left(w_{1}\right)\right)^{t_{j}}$ and bstag must be equal to $K_{T}\left[I\left(w_{1}\right)\right] / K_{T}\left[I_{1}\right]$. Note that $G_{6}$ does not use the $K_{T}$ key array in any other way except in this test. Therefore, since $K_{T}$ component keys are all random in $Z_{p}^{*}$, and the game makes polynomially-many such tests, the probability that such test ever succeeds for $I\left(w_{1}\right) \neq I_{1}$ is negligible, and hence $p_{6} \approx p_{5}$.

Game $G_{7}$. We add an abort if $A$ ever invokes two instances of Search protocol which involve the same env ciphertext but two different values bstag and bstag' s.t. there exists two keywords $w_{1}, w_{1}^{\prime}$ s.t. $(\text { bstag })^{1 / \rho_{1}}=\left(H\left(w_{1}\right)\right)^{K_{T}\left[I_{1}\right]}$ and $\left(\text { bstag }^{\prime}\right)^{1 / \rho_{1}}=\left(H\left(w_{1}^{\prime}\right)\right)^{K_{T}\left[I_{1}\right]}$. Use the notation $t_{j}$ for $K_{T}\left[I_{1}\right] \cdot \rho_{1}$ used in the $j$-th GenToken instance as in game $G_{6}$. Note that the above condition implies that bstag $=\left(H\left(w_{1}\right)\right)^{t_{j}}$ and bstag $^{\prime}=\left(H\left(w_{1}^{\prime}\right)\right)^{t_{j}}$ if ciphertext env was generated by the $j$-th instance of GenToken. It is easy to see that the probability of encountering such two pairs of values $\left(w_{1}\right.$, bstag) and $\left(w_{1}^{\prime}\right.$, bstag $\left.^{\prime}\right)$ must be negligible under the OM-GDH assumption if $H$ is modeled as a random oracle, hence $p_{7} \approx p_{6}$.

Let $\epsilon$ be the probability of the above event. The reduction on the input a OM-GDH challenge $h_{1}, h_{2}$, emulates game $G_{3}$ except that on each $A$ 's query $w$ to $H$, it picks random $r_{1}, r_{2}$ in $Z_{p}^{*}$ and sets $H(w) \leftarrow\left(h_{1}\right)^{r_{1}}\left(h_{2}\right)^{r_{2}}$. Furthermore, the reduction picks a random index $j$ between 1 and the maximum number of GenToken instances $A$ can invoke, and when servicing the $j$-th instance of GenToken, the reduction sends the $a_{1}$ value in this instance to the OM-GDH challenger, who replies with $b_{1} \leftarrow\left(a_{1}\right)^{t}$ for $t$ chosen by the OM-GDH challenge game. The reduction passes this $b_{1}$ in its response to $A$. Finally, for each Search protocol instance on which $A$ sends ciphertext env, the reduction takes bstag sent by $A$ along with env, and for each query $w$ made by $A$ to $H$ it consults the DDH oracle if $\left(a_{1}, b_{1}, H(w)\right.$, bstag) is a DDH tuple. If the reduction finds two instances of Search on which the above check verifies, one for $\left(w\right.$, bstag) and the other for $\left(w^{\prime}\right.$, bstag $\left.^{\prime}\right) \neq(w$, bstag $)$, it computes $h_{1}^{t}$ and $h_{2}^{t}$ as, respectively, (bstag $\left.^{r_{2}}\left(\text { bstag }^{\prime}\right)^{-r_{2}}\right)^{1 /\left(r_{1} r_{2}^{\prime}-r_{1}^{\prime} r_{2}\right)}$ and $\left(\text { bstag }^{r_{1}}\left(\text { bstag }^{\prime}\right)^{-r_{1}}\right)^{1 /\left(r_{1}^{\prime} r_{2}-r_{1} r_{2}^{\prime}\right)}$, where $H(w)=\left(h_{1}\right)^{r_{1}}\left(h_{2}\right)^{r_{2}}$ and $H\left(w^{\prime}\right)=\left(h_{1}\right)^{r_{1}^{\prime}}\left(h_{2}\right)^{r_{2}^{\prime}}$. The probability of reduction's success is $(1 / m) \cdot \epsilon$ where $m$ is the upper-bound on the number of GenToken instances $A$ invokes, minus the negligible probability that $r_{1} r_{2}^{\prime}=r_{1}^{\prime} r_{2}$.

Game $G_{8}$. We replace the $\operatorname{PRF} F_{p}\left(K_{I}, \cdot\right)$ with a random function $F_{I}(\cdot)$ onto $Z_{p}^{*} \cdot p_{8} \approx p_{7}$ by the PRF property of $F_{p}$.

Game $G_{9}$. We modify the way $G_{8}$ processes the Search procedure as follows: Once $G_{9}$ identifies $w_{1}$ given bstag and the $\left(I_{1}, \rho_{1}\right)$ in QList(env) as described above, it computes strap $\leftarrow F_{G}\left(K_{s}, w_{1}\right)$ and $\left(K_{z}, K_{e}\right) \leftarrow\left(F_{\tau}(\operatorname{strap}, 1), F_{\tau}(\right.$ strap, 2$\left.)\right)$. Then, for each $c$, $G_{9}$ computes $z_{c} \leftarrow F_{p}\left(K_{z}, c\right)$, and given $x \operatorname{token}[c]=(\operatorname{xtoken}[c, 2], \ldots, x \operatorname{token}[c, n])$, for each $i=2, \ldots, n, G_{9}$ searches for $w_{i}$ in W s.t. xtoken $[c, i]=\left(F_{G}\left(K_{X}, w_{i}\right)\right)^{z_{c} \cdot \rho_{i}}$ and $\operatorname{ind}_{c} \in \operatorname{DB}\left(w_{i}\right)$, where $\operatorname{ind}_{c}$ corresponds to the $c$-th tuple $(\mathrm{e}, y)$ in $\mathbf{t}=\mathbf{T}\left[w_{1}\right]$. If $G_{9}$ finds such $w_{i}$ for all $i$ then it sends e to $A$.

Let us use the notation $W$ (ind) for the set of keywords in record ind, i.e. the set of $w$ 's in $\mathrm{W}$ s.t. ind $\in \mathrm{DB}(w)$. Note that if the above check succeeds for any $c, i$ in game $G_{9}$ then it must also succeed in game $G_{8}$, because if xtoken $[c, i]=\left(F_{G}\left(K_{X}, w_{i}\right)\right)^{z_{c} \cdot \rho_{i}}$ then 
(xtoken $[c, i])^{y_{c} / \rho_{i}}$ is equal to $\left(F_{G}\left(K_{X}, w_{i}\right)\right)^{\text {xind }_{c}}$, and if $\operatorname{ind}_{c} \in \mathrm{DB}\left(w_{i}\right)$ then this xtrap value is included in set XSet. Hence the only difference between $G_{9}$ and $G_{8}$ can occur if for some $c, i$ we have that $(x \operatorname{xtoken}[c, i])^{y_{c} / \rho_{i}} \in \mathrm{XSet}$ but xtoken $[c, i] \neq\left(F_{G}\left(K_{X}, w\right)\right)^{z_{c} \cdot \rho_{i}}$ for all $w \in W\left(\right.$ ind $\left._{c}\right)$.

Let us denote exponent $K_{X}\left[I_{i}\right] \cdot \rho_{i}$ used in exponentiating $a_{i}$ in the $j$-th GenToken instance as $t_{i, j}$. Clearly, $G_{8}$ can pick $t_{i, j}$ 's at random in $Z_{p}^{*}$ and define the corresponding $\rho_{i}$ value as $t_{i, j} / K_{X}\left[I_{i}\right]$. Using this notation, the event that (xtoken $\left.[c, i]\right)^{y_{c} / \rho_{i}}$ matches some value in XSet is equivalent to existence of some ind ${ }^{*}$ and $w^{*} \in W\left(\right.$ ind $\left.^{*}\right)$ s.t.

$$
(\times \operatorname{token}[c, i])^{1 / z_{c}}=\left(H\left(w^{*}\right)\right)^{t_{i, j} \cdot \frac{K_{x}\left[I\left(w^{*}\right)\right]}{K_{x}\left[I_{i}\right]} \cdot \frac{F_{I}\left(\text { ind }^{*}\right)}{F_{I}\left(\text { ind }_{c}\right)}}
$$

At the same time, the constraint that $x \operatorname{token}[c, i] \neq\left(F_{G}\left(K_{x}, w\right)\right)^{z_{c} \cdot \rho_{i}}$ for all $w \in W\left(\right.$ ind $\left._{c}\right)$ implies that for all $w \in W\left(\right.$ ind $\left._{c}\right)$ we have

$$
(\text { token }[c, i])^{1 / z_{c}} \neq(H(w))^{t_{i, j} \cdot \frac{K_{x}[I(w)]}{K_{x}\left[I_{i}\right]}}
$$

We will argue that there is at most negligible probability that equation (1) holds while equation (2) also holds for every $w \in W\left(\right.$ ind $\left._{c}\right)$. It will follow that $p_{9} \approx p_{8}$.

Observe that if equation (1) holds for ind ${ }^{*}=\operatorname{ind}_{c}$ and $I\left(w^{*}\right)=I_{i}$, then $(x \operatorname{token}[c, i])^{1 / z_{c}}=$ $\left(H\left(w^{*}\right)\right)^{t_{i, j}}$ for $w^{*} \in W\left(\right.$ ind $\left._{c}\right)$, but this contradicts equation (2), which in the case $I\left(w^{*}\right)=I_{i}$ implies that $(x \operatorname{token}[c, i])^{1 / z_{c}} \neq\left(H\left(w^{*}\right)\right)^{t_{i, j}}$. Below we argue that equation (1) can hold only with negligible probability for either if ind* $\neq \operatorname{ind}_{c}$ or if $I\left(w^{*}\right) \neq I_{i}$, which together implies that the two equations can hold together only with negligible probability.

(Case a:) We argue that equation (1) can hold for ind $\neq$ ind $_{c}$ with at most negligible probability. Let us modify game $G_{8}$ into $G^{\prime}$ s.t. $G^{\prime}$ does not append pair (e,y) into the $\mathbf{T}[w]$ tuple list, but instead appends triples (e, ind, $z$ ), i.e. in particular $G^{\prime}$ does not query $F_{I}$ in creating the $\mathbf{T}$ lists. Secondly, game $G^{\prime}$ also does not create the XSet data structure during EDBSetup. Instead, it modifies the test procedure performed by $G_{8}$ for each $c$ and $i$ in the Search protocol: Instead of checking whether (xtoken $[c, i])^{\left(y_{c} / \rho_{i}\right)}$ is in the XSet, $G^{\prime}$ searches for $w^{*} \in \mathrm{W}$ and ind ${ }^{*} \in \mathrm{DB}(w)$ s.t. equation (1) holds, using the ind ${ }_{c}, z_{c}$ values kept in the $c$-th tuple in $\mathbf{T}\left[w_{1}\right]$. As we argued above, these are equivalent conditions, and therefore $G^{\prime}$ provides an identical view as $G_{8}$. Note furthermore that the test in equation (1) can be implemented by testing whether $a=b^{F_{I}\left(\text { ind }^{*}\right) / F_{I}\left(\text { ind }_{c}\right)}$ where $a=(\operatorname{xtoken}[c, i])^{1 / z_{c}}$ and $b=\left(H\left(w^{*}\right)\right)^{t_{i, j} \cdot\left(K_{X}\left[I\left(w^{*}\right)\right] / K_{X}\left[I_{i}\right]\right)}$. In other words, game $G^{\prime}$ can operate with an access to $F_{I}$ restricted as follows: $G^{\prime}$ specifies a tuple $\left(a, b, x, x^{*}\right)$ and the oracle returns 1 if $a^{F_{I}(x)}=b^{F_{I}\left(x^{*}\right)}$, and 0 otherwise. Since $H$ is a random function with range $G$, we have that except for negligible probability all $\left(a, b, x, x^{*}\right)$ queries of $G^{\prime}$ involve $b \neq 1$. Since $F_{I}$ is a random function onto $Z_{p}^{*}$ and game $G^{\prime}$ makes polynomially-many such queries, there is only a negligible probability that the oracle returns 1 for any query s.t. $x \neq x^{*}$, which implies that there is at most negligible probability that equation (1) holds for ind $\neq \neq$ ind.

(Case b:) Using a similar reasoning, we argue that equation (1) can hold for $I\left(w^{*}\right) \neq I_{i}$ also with at most negligible probability. Note that the only time game $G^{\prime}$ accesses key array 
$K_{X}$ is also in testing equation (1), which is equivalent to specifying a query $\left(a, b, I, I^{*}\right)$ for $a=(\operatorname{xtoken}[c, i])^{\left(1 / z_{c}\right) \cdot F_{I}\left(\text { ind }_{c}\right)}, b=\left(H\left(w^{*}\right)\right)^{t_{i, j} \cdot F_{I}\left(\text { ind }^{*}\right)}, I=I_{i}$, and $I^{*}=I\left(w^{*}\right)$, and receiving back 1 if $a^{K_{X}[I]}=b^{K_{X}\left[I^{*}\right]}$. Note that probability that $b=1$ is negligible because $H$ is a random function onto $G$. Since the individual keys in array $K_{X}$ are independently chosen in $Z_{p}^{*}$ and $b G^{\prime}$ makes polynomially many such queries, it follows that there is a negligible probability that any such query succeeds for $I \neq I^{*}$, i.e. for $I_{i} \neq I\left(w^{*}\right)$.

Game $G_{10}$. We modify the test game $G_{9}$ uses to identify $w_{i}$ given xtoken $[c, i]$ and $z_{c}, \rho_{i}$ by amending verification that xtoken $[c, i]=\left(F_{G}\left(K_{X}, w_{i}\right)\right)^{z_{c} \cdot \rho_{i}}$ and $\operatorname{ind}_{c} \in \mathrm{DB}\left(w_{i}\right)$ with the additional constraint that $I\left(w_{i}\right)=I_{i}$ for $I_{i}$ is retrieved from QList(env). The event that differentiates the two games is that xtoken $[c, i]=\left(H\left(w_{i}\right)\right)^{K_{X}\left[I\left(w_{i}\right)\right] \cdot z_{c} \rho_{i}}$ for ind $_{C} \in$ $\mathrm{DB}\left(w_{i}\right)$ but $I\left(w_{i}\right) \neq I_{i}$. We argue that this event occurs with negligible probability, and hence $p_{10} \approx p_{9}$, even against an all-powerful adversary. Using the notation $t_{i, j}$ for $K_{X}\left[I_{i}\right] \cdot \rho_{i}$ used on the $j$-th GenToken session, the above equation can be rewritten as xtoken $[c, i]=\left(H\left(w_{i}\right)\right)^{z_{c} \cdot t_{i, j} \cdot \frac{K_{X}\left[I\left(w_{i}\right)\right]}{K_{X}\left[I_{i}\right]}}$. In particular, the discrete logarithm between xtoken $[c, i]$ and $\left(H\left(w_{i}\right)\right)^{z_{c} \cdot t_{i, i}}$ must be equal to $K_{X}\left[I_{i}\right] / K_{X}\left[I\left(w_{i}\right)\right]$. Note the $G_{10}$ does not use the key array $K_{X}$ in any other way except in this test. Therefore, since $K_{X}$ component keys are random in $Z_{p}^{*}$, and the game makes polynomially-many such tests, the probability that such test ever succeeds for $I\left(w_{i}\right) \neq I_{i}$ is negligible, and hence $p_{10} \approx p_{9}$.

Game $G_{11}$. We add an abort if the game ever encounters a ciphertext env, index $i$, a pair of counters $c, c^{\prime}$ and two keywords $w_{/}=w_{i}^{\prime}$ s.t. xtoken $[c, i]=\left(H\left(w_{i}\right)\right)^{K_{X}\left[I_{i}\right] \cdot z_{c} \cdot \rho_{i}}$ and xtoken $\left[c^{\prime}, i\right]=\left(H\left(w_{i}^{\prime}\right)\right)^{K_{X}\left[I_{i}\right] \cdot z_{c^{\prime}} \cdot \rho_{i}}$. We intend this abort to include any Search session that some ciphertext env is used, including the case that this "collision" occurs on two different Search sessions using the same env for the same counter $c^{\prime}=c$. Using the $t_{i, j}$ notation for $K_{X}\left[I_{1}\right] \cdot \rho_{i}$ used in the $j$-th session of GenToken, we rewrite this as xtoken $[c, i]^{1 / z_{c}}=$ $\left(H\left(w_{i}\right)\right)^{t_{i, j}}$ and xtoken $\left[c^{\prime}, i\right]^{1 / z_{c^{\prime}}}=\left(H\left(w_{i}^{\prime}\right)\right)^{t_{i, j}}$. It is easy to see that the probability of encountering such two tuples $\left(w_{i}\right.$, xtoken $\left.[c, i], z_{c}\right)$ and $\left(w_{i}^{\prime}\right.$, xtoken $\left.\left[c^{\prime}, i\right], z_{c^{\prime}}\right)$ for $w_{i} \neq w_{i}^{\prime}$ must be negligible under the OM-GDH assumption in the ROM model for $H$, and hence $p_{11} \approx p_{10}$.

The reduction is essentially the same as the one given in the argument for game $G_{7}$. Let $\epsilon$ be the probability of the above event. The reduction on the input a OM-GDH challenge $h_{1}, h_{2}$, emulates game $G_{11}$ except that on each $A$ 's query $w$ to $H$, it picks random $r_{1}, r_{2}$ in $Z_{p}^{*}$ and sets $H(w) \leftarrow\left(h_{1}\right)^{r_{1}}\left(h_{2}\right)^{r_{2}}$. The reduction also picks a random index $j$ between 1 and the maximum number of GenToken instances $A$ can invoke, and when servicing the $j$-th instance of GenToken, the reduction picks $i$ at random between 2 and $n$, sends the $a_{i}$ value in this GenToken instance to the OM-GDH challenger, who replies with $b_{i} \leftarrow\left(a_{i}\right)^{t}$ for the exponent $t$ chosen in the OM-GDH challenge game. The reduction passes this $b_{i}$ in its response to $A$. Finally, for each Search protocol instance on which $A$ sends ciphertext env, for each $c$, the reduction takes xtoken $[c, i]$ sent by $A$ along with env, and for each query $w$ made by $A$ to $H$ it consults the $\mathrm{DDH}$ oracle if $\left(a_{1}, b_{1}, H(w),(\operatorname{xtoken}[c, i])^{1 / z_{c}}\right)$ is a DDH tuple. If the reduction finds two such instances on which the above check verifies, one for $\left(w, x \operatorname{token}[c, i], z_{c}\right)$ and the other for $\left(w^{\prime}, x \operatorname{token}\left[c^{\prime}, i\right], z_{c^{\prime}}\right)$ s.t. $w \neq w^{\prime}$, it 
computes $h_{1}^{t}$ and $h_{2}^{t}$ as, respectively, $\left((\operatorname{xtoken}[c, i])^{r_{2} / z_{c}}\left(\operatorname{xtoken}\left[c^{\prime}, i\right]\right)^{-r_{2} / z_{c^{\prime}}}\right)^{1 /\left(r_{1} r_{2}^{\prime}-r_{1}^{\prime} r_{2}\right)}$ and $\left((x \operatorname{token}[c, i])^{r_{1} / z_{c}}\left(\text { xtoken }\left[c^{\prime}, i\right]\right)^{-r_{1} / z_{c^{\prime}}}\right)^{1 /\left(r_{1}^{\prime} r_{2}-r_{1} r_{2}^{\prime}\right)}$, where $H(w)=\left(h_{1}\right)^{r_{1}}\left(h_{2}\right)^{r_{2}}$ and $H\left(w^{\prime}\right)=$ $\left(h_{1}\right)^{r_{1}^{\prime}}\left(h_{2}\right)^{r_{2}^{\prime}}$. The probability of reduction's success is $(1 /(m n)) \cdot \epsilon$ where $m$ is the upperbound on the number of GenToken instances $A$ invokes and $n$ an upper-bound on the length of a conjunctive query, minus the negligible probability that $r_{1} r_{2}^{\prime}=r_{1}^{\prime} r_{2}$.

Game $G_{12}$. Note that in game $G_{11}$ there is at most one vector $\bar{w}=\left(w_{1}, \ldots, w_{n}\right)$ of keywords the game finds in all Search protocol instances for a given ciphertext env which the game produced in some GenToken protocol instance. This is because from game $G_{7}$ on, the game finds at most one $w_{1}$ corresponding to any env, and from game $G_{11}$ on, the game also finds at most one $w_{i}$ corresponding to any env and $i$ between 2 and the index $n$ used in the GenToken session which produced env. This unique query $\bar{w}$ corresponding to env should be thought of as the effective query $A$ made in the GenToken instance which produced the env. Note, moreover, that av $(\bar{w})$ is equal to the vector of attributes $\left(I_{1}, \ldots, I_{n}\right)$ which $A$ specified in that GenToken instance, and therefore the only way this instance produced a ciphertext env is if $\left(I_{1}, \ldots, I_{n}\right) \in \mathrm{P}$. Note finally, that the only ciphertext e the game $G_{11}$ supplies to $A$ correspond to indexes $\operatorname{ind}_{c}$ in $\mathrm{DB}\left(w_{1}\right)$ s.t. $\operatorname{ind}_{c} \in \mathrm{DB}\left(w_{i}\right)$ for $i=2, \ldots, n$, i.e. for ind 's in $\mathrm{DB}(\bar{w})$.

These observations lead us to the new game $G_{12}$, a modification of $G_{11}$, which operates as follows. Game $G_{12}$ does not create either TSet or XSet data structure in EDBSetup, only picks keys $K_{S}, F_{X}, K_{T}, K_{P}, K_{M}$ (it skips choosing key $K_{I}$, which it will not need). Game $G_{12}$ services the GenToken instances as $G_{11}$, but on calls to Search, when it finds counter $c$ s.t. xtoken $\left.[c, i]=F_{G}\left(K_{X}, w_{i}\right)\right)^{z_{c} \cdot \rho_{i}}$, ind ${ }_{c} \in \mathrm{DB}\left(w_{i}\right)$, and $I\left(w_{i}\right)=I_{i}$, for all $i=2, \ldots, n$, it assembles query $\bar{w}$ from the identified $w_{1}, \ldots, w_{n}$ terms, and sends it to I-SSE $_{\mathcal{L}}(\mathrm{DB}, \mathrm{RDK}, \mathrm{P})$. Since $\operatorname{av}(\bar{w})=\left(I_{1}, \ldots, I_{n}\right) \in \mathrm{P}$, it receives back $\left(\operatorname{DB}(\bar{w}), \operatorname{RDK}(\operatorname{DB}(\bar{w}))\right.$, Mask $\left.\left(\left|\operatorname{DB}\left(w_{1}\right)\right|\right)\right)$. Game $G_{12}$ then assigns the indexes in $\mathrm{DB}(\bar{w})$ to random indexes between 1 and $\operatorname{Mask}\left(\left|\mathrm{DB}\left(w_{1}\right)\right|\right)$, unless some of these were previously assigned. To keep track of previously seen indexes, $G_{12}$ keeps a list TList, indexed by $w_{1}$ 's, s.t. TList $\left(w_{1}\right)$ stores tuples $\left(c\right.$, ind, e) s.t. counter $c$ in $\mathbf{T}\left[w_{1}\right]$ was assigned during some Search instance to index ind, and the corresponding ciphertext was created as e. Initially TList $\left(w_{1}\right)$ is empty for all $w_{1} \in \mathbf{W}$, but when $G_{12}$ identifies the query $\bar{w}=\left(w_{1}, \ldots, w_{n}\right)$ in the Search protocol, sends it to I-SSE $_{\mathcal{L}}$, and receives the tuple $\left(\operatorname{DB}(\bar{w}), \operatorname{RDK}(\operatorname{DB}(\bar{w})), \operatorname{Mask}\left(\left|\operatorname{DB}\left(w_{1}\right)\right|\right)\right), G_{8}$ checks if for any ind $\in \operatorname{DB}(\bar{w})$ the ind value was already in the TList $\left(w_{1}\right)$. For all ind's in $\operatorname{DB}(\bar{w})$ which are not in TList $\left(w_{1}\right), G_{8}$ assigns them to a random subset of remaining counters $c$ between 1 and TSetL, and for each $(c$, ind) pair it computes a corresponding ciphertext e using the $K_{e}$ key computed using $K_{S}$ from $w_{1}$ as in game $G_{9}$ above, setting rind $\leftarrow P_{\tau}\left(K_{P}\right.$, ind $)$, rdk $\leftarrow \operatorname{RDK}($ ind $)$, and $\mathrm{e} \leftarrow \operatorname{Enc}\left(K_{e},(\right.$ rind $\left.\mid r d k)\right)$.

In this way $G_{12}$ creates a view which the adversary gets of some T-set TSet $\left(w_{1}\right)$ throughout its interaction with the MC-OXT scheme, and this view matches that created by $G_{11}$, where the assignment between indexes ind $\in \mathrm{DB}\left(w_{1}\right)$ and the counters in $\left\{1, \ldots, \operatorname{Mask}\left(\left|\mathrm{DB}\left(w_{1}\right)\right|\right)\right\}$ is done during the EDBSetup procedure, because in both cases this assignment is random. The full description of game $G_{12}$ is shown in Figure 3 as a code of a simulator algorithm $S$, broken down into three subprocesses $S_{0}, S_{1}, S_{2}$, servicing respectively EDBSetup, GenToken, 
and Search protocols. It follows that $p_{12}=p_{11}$.

This completes the proof of security because it concludes the argument that there is at most negligible difference between $p_{0}$, which is the probability that $\operatorname{Real}_{A}^{\Pi}(\lambda)$ outputs 1 , and $p_{12}$, which is the probability that $\operatorname{Ideal}_{A, S}^{\Pi}(\lambda)$ outputs 1 . Moreover, the simulator $S$ maintains the constraint demanded by the security definition that the number of unique queries $\bar{w}$ it makes to I-SSE$_{\mathcal{L}}$ does not exceed the number of GenToken instances which adversary $A$ makes.

\section{B OSPIR-OXT Instantiated with Hashed-DH OPRF}

In Figure 4 we present the instantiation of protocol OSPIR-OXT with the Hashed-DH OPRF described at the end of Section 4.1. It serves as an illustration of a concrete realization of the protocol and it is the basis for our analysis and implementation. We note some notational differences relative to the abstract OSPIR-OXT protocol that arise from the specific HashedDH instantiation: We denote keys $K_{T}$ and $K_{X}$ of PRF $F_{G}^{m}$ by vectors of exponents in $Z_{p}^{*}$, respectively $\left(k_{1}, \ldots, k_{m}\right)$ and $\left(e_{1}, \ldots, e_{m}\right)$, where $m$ is the number of attributes. Also, because of the specific OPRF instantiation we equate $a_{s}$ to $a_{1}$ in $\mathcal{C}$ 's message of the GenToken protocol, instead of computing these two blinded versions of keyword $w_{1}$ separately, as in Figure 2. 
Group operations. $G$ is a cyclic group of prime order $p$ generated by an element $g . H$ is a hash function with range in $G \backslash\{1\}$.

\section{EDBSetup(DB, RDK)}

Key Generation. $\mathcal{D}$ picks key $K_{S}$ and two vectors of elements $K_{T}=\left(k_{1}, \ldots, k_{m}\right)$ and $K_{X}=\left(e_{1}, \ldots, e_{m}\right)$ at random in $Z_{p}^{*}\left(m=\right.$ number of attributes); key $K_{I}$ for PRF $F_{p}$; and key $K_{M}$ for a symmetric authenticated encryption AuthEnc. $F_{p}$ and $F_{\tau}$ are PRF's which outputs strings in respectively $Z_{p}^{*}$ and $\{0,1\}^{\tau}$, and $\tau$ is a security parameter.

- Initialize XSet to an empty set, and initialize $\mathbf{T}$ to an empty array indexed by group elements in $G$.

- For each $w=(i, v a l) \in \mathbf{W}$, build the tuple list $\mathbf{t}$ and add elements to set XSet as follows:

- Initialize $\mathbf{t}$ to an empty list.

- Set strap $\leftarrow(H(w))^{K_{S}}, \quad$ stag $\leftarrow(H(w))^{k_{i}}\left[=F_{G}^{m}\left(K_{T}, w\right)\right], \quad\left(K_{z}, K_{e}\right) \leftarrow\left(F_{\tau}(\right.$ strap, 1$), F_{\tau}($ strap, 2$\left.)\right)$.

- Initialize $c \leftarrow 0$; then for all ind in $\mathrm{DB}(w)$ in random order:

$*$ Set $r d k \leftarrow \operatorname{RDK}($ ind $), \mathrm{e} \leftarrow \operatorname{Enc}\left(K_{e}\right.$, (ind $\left.\left.\mid r d k\right)\right)$, xind $\leftarrow F_{p}\left(K_{I}\right.$, ind).

$*$ Set $c \leftarrow c+1, z_{c} \leftarrow F_{p}\left(K_{z}, c\right), y \leftarrow$ xind $\cdot z_{c}^{-1}$. Append $(\mathrm{e}, y)$ to $\mathbf{t}$.

* Set $\times \operatorname{tag} \leftarrow H(w)^{e_{i} \cdot \text { xind }}\left[=\left(F_{G}^{m}\left(K_{X}, w\right)\right)^{\text {xind }}\right]$ and add xtag to XSet.

$-\mathbf{T}[\operatorname{stag}] \leftarrow \mathbf{t}$.

- Create TSet $\leftarrow$ TSetSetup' $(\mathbf{T})$, and output key $K=\left(K_{S}, K_{X}, K_{T}, K_{I}, K_{M}\right)$ and EDB $=\left(\right.$ TSet, XSet, $\left.K_{M}\right)$.

\section{GenToken protocol}

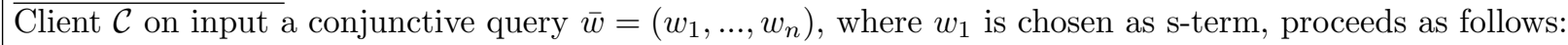

- Pick $r_{1}, \ldots, r_{n} \stackrel{\$}{\leftarrow} Z_{p}^{*}$ and set $a_{j} \leftarrow\left(H\left(w_{j}\right)\right)^{r_{j}}$ for $j=1, \ldots, n$.

- Send to $\mathcal{D}$ the blinded queries $a_{1}, \ldots, a_{n}$ and the attribute sequence av $=\left(I\left(w_{1}\right), \ldots, I\left(w_{n}\right)\right)$.

Data owner $\mathcal{D}$ on input policy $P$ and key $K$ proceeds as follows:

- Abort if av $\notin P$. Otherwise set av as a local output. Pick $\rho_{1}, \ldots, \rho_{n} \stackrel{\$}{\leftarrow} Z_{p}^{*}$.

- Set strap ${ }^{\prime} \leftarrow\left(a_{1}\right)^{s}$, bstag $^{\prime} \leftarrow\left(a_{1}\right)^{k_{i_{1}} \cdot \rho_{1}}\left[=\left(a_{1}\right)^{K_{T}\left[i_{1}\right] \cdot \rho_{1}}\right]$, and bxtrap ${ }_{j}^{\prime} \leftarrow\left(a_{j}\right)^{e_{i_{j}} \cdot \rho_{j}}\left[=\left(a_{j}\right)^{K_{X}\left[i_{j}\right] \cdot \rho_{j}}\right]$ for $j=2, \ldots, n$.

- Reply to $\mathcal{C}$ with $\left(\right.$ strap $^{\prime}$, bstag $^{\prime}$, bxtrap $_{2}^{\prime}, \ldots$, bxtrap $\left._{n}^{\prime}\right)$ and env $=\operatorname{AuthEnc}_{K_{M}}\left(\rho_{1}, \ldots, \rho_{n}\right)$.

$\mathcal{C}$ sets:

- strap $\leftarrow\left(\text { strap }^{\prime}\right)^{r_{1}^{-1}} ;$ bstag $\leftarrow\left(\text { bstag }^{\prime}\right)^{r_{1}^{-1}} ;$ bxtrap $_{j} \leftarrow\left(\text { bxtrap }_{j}^{\prime}\right)^{r_{j}^{-1}}\left(\forall_{j=2, \ldots, n}\right)$;

- token $\leftarrow$ (env, strap, bstag, bxtrap $_{2}, \ldots$, bxtrap ${ }_{n}$ ).

Search protocol

Client $\mathcal{C}$ on input token proceeds as follows:

- Set $\left(K_{z}, K_{e}\right) \leftarrow F\left(_{\tau}(\right.$ strap, 1$), F($ strap, 2$\left.)\right)$; send to $\mathcal{E}$ the message (env, bstag, bxtoken[1], bxtoken[2], ..) defined as:

- For $c=1,2, \ldots$, until $\mathcal{E}$ sends stop:

* Set $z_{c} \leftarrow F_{p}\left(K_{z}, c\right)$ and set bxtoken $[c, i] \leftarrow\left(\operatorname{bxtrap}_{i}\right)^{z_{c}}$ for $i=2, \ldots, n$.

* Set bxtoken $[c] \leftarrow$ (bxtoken $[c, 2], \ldots$, bxtoken $[c, n])$.

Server $\mathcal{E}$ on input EDB $=\left(\right.$ TSet $\left., \mathrm{XSet}, K_{M}\right)$ responds as follows:

- Upon receiving env, bstag from $\mathcal{C}$, decrypt/verify env; if verification fails return "no results" and stop.

- Set stag $\leftarrow$ (bstag $)^{1 / \rho_{1}}$ and retrieve $\mathbf{t} \leftarrow$ TSetRetrieve $(T S e t$, stag) from TSet.

- For $c=1, \ldots,|\mathbf{t}|$ do:

- Receive bxtoken $[c]$ from $\mathcal{C}$ and retrieve value $(\mathrm{e}, y)$ from the $c$-th tuple in $\mathbf{t}$.

- Check if bxtoken $[c, i]^{y / \rho_{i}} \in \mathrm{XSet}$ for all $i=2, \ldots, n$. If so, send e to $\mathcal{C}$ (else nothing is returned for this tuple).

- When last tuple in $\mathbf{t}$ is reached, send stop to $\mathcal{C}$ and halt.

For each received e client $\mathcal{C}$ computes (ind $\mid r d k) \leftarrow \operatorname{Dec}\left(K_{e}, e\right)$ and outputs (ind, rdk). 